\title{
SUPERHEATING FIELD OF TYPE II SUPERCONDUCTORS*
}

\author{
S. JONATHAN CHAPMAN ${ }^{\dagger}$
}

\begin{abstract}
The superheating magnetic field of a type II superconductor is examined, using the time-dependent Ginzburg-Landau equations and the methods of formal asymptotics. The superconducting solution in a halfspace is found to exist only for magnetic fields lower than some critical value where there is a folding over of the solution branch. A linear stability analysis is performed both in one and two dimensions, giving differing criteria for stability. Finally, superheating fields for more general geometries are considered, and in particular the case of a sine-wave perturbation of a halfspace is examined.
\end{abstract}

Key words. superconductivity, Ginzburg-Landau equations, superheating field

AMS subject classifications. $82 \mathrm{D} 55$

1. Introduction. This paper is concerned with the response of a superconducting material to an externally imposed magnetic field. This response is most conveniently described by Fig. 1, which shows the preferred state of the superconductor as a function of $H_{0}$, the applied magnetic field, and $\kappa$, a material parameter (known as the Ginzburg-Landau parameter) which determines the type of superconducting material; $\kappa<1 / \sqrt{2}$ describes what is known as a type I superconductor, and $\kappa>1 / \sqrt{2}$ describes what is known as a type II superconductor.

For type I superconductors there is a critical magnetic field $H_{c}$ (known as the thermodynamic critical field) below which the material will be in the superconducting state but above which it will revert to the normally conducting (normal) state. The transition between normal and superconducting states as the applied magnetic field is raised or lowered through $H_{c}$ takes place by means of phase boundaries separating normal regions from superconducting regions sweeping through the material and is described in [7], [10], and [17].

For type II superconductors a third state exists, which is known as the mixed state. The mixed state, as its name suggests, is neither wholly superconducting nor wholly normal but consists of many normal filaments embedded in a superconducting matrix. Each of these filaments carries with it a quantised amount of magnetic flux and is circled by a vortex of superconducting current; thus these filaments are often known as vortices. The transition from the normal state to the mixed state takes place via a bifurcation as the magnetic field is lowered through some critical value $H_{c_{2}}$ (known as the upper critical field) and is described in [1], [6], [8], [9], [18], [21], and [23]. This bifurcation is subcritical for type I superconductors but supercritical for type II superconductors, hence the observation of the mixed state only for type II superconductors. There is a mutual repulsion between superconducting vortices, which leads to the formation of a hexagonal lattice of vortices in the mixed state, as observed in [12].

The transition between superconducting and mixed states is less well studied mathematically, and this will be the subject of the present paper. The critical field $H_{c_{1}}$ plotted in Fig. 1 (known as the lower critical field) is calculated on the basis of an energy argument; it is the field at which the energy of the wholly superconducting solution becomes equal to the energy of the single vortex solution for an infinite

* Received by the editors August 30, 1993; accepted for publication (in revised form) September 6,1994 .

$\dagger$ Mathematical Institute, University of Oxford, 24-29 St. Giles', Oxford OX1 3LB, United Kingdom. 


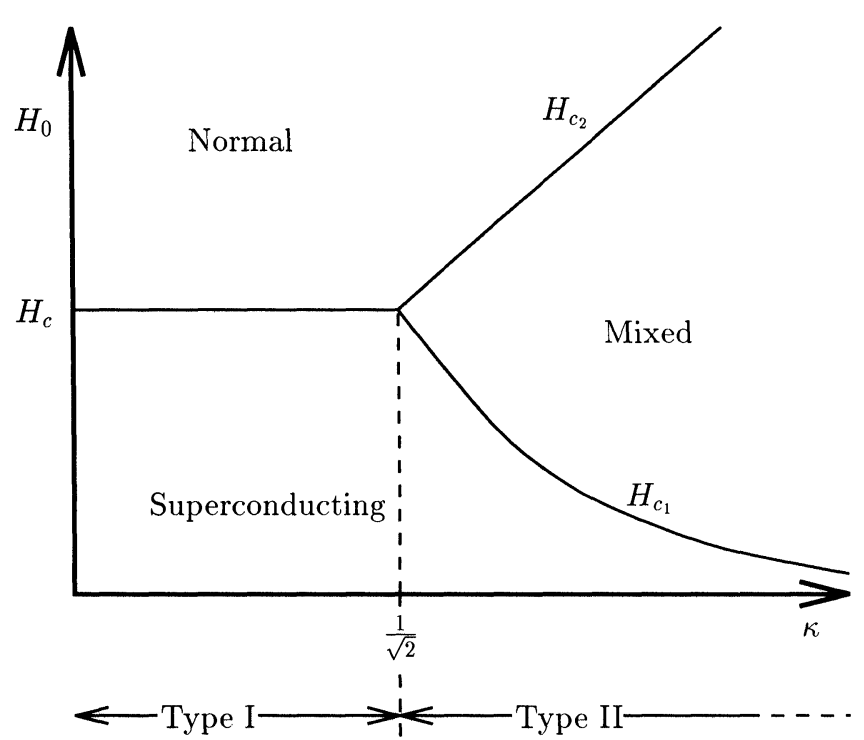

FIG. 1. The response of a superconductor as a function of the applied magnetic field and the Ginzburg-Landau parameter $\kappa$.

superconductor. In fact, as $H_{0}$ is raised, there is a barrier to the generation of vortices in a superconductor, and there exists a "superheating field" $H_{s h}$ such that for $H_{c_{1}}<$ $H_{0}<H_{s h}$ the superconducting solution is still locally stable, even though it is not the global minimum energy solution. ${ }^{1}$ We will find that the response of a superconductor is different in two dimensions than in one dimension, leading to a different value of $H_{s h}$ from that obtained by de Gennes [14], Matricon and Saint-James [20], and Fink [13]. We note that Bean and Livingston have also calculated approximate surface barrier fields, using an energy argument [3]. The approach we adopt is via the timedependent Ginzburg-Landau equations (to be introduced in the next section) and again leads to a different result.

The results we obtain do agree with that obtained for a halfspace by Kramer [19], who uses the steady-state Ginzburg-Landau equations with the positivity of the second variation of the free energy being the criterion for stability. We will see that the present approach allows the superheating field of an arbitrary body to be calculated, including the site of first instability.

2. The Ginzburg-Landau model. For a more complete introduction to the Ginzburg-Landau theory of superconductivity the reader is referred to [10] and [11] and the references therein. Here we merely state the dimensionless, time-dependent Ginzburg-Landau equations as

$$
\begin{gathered}
-\frac{\alpha}{\kappa^{2}} \frac{\partial \Psi}{\partial t}-\frac{\alpha i}{\kappa} \Psi \phi+\left(\frac{1}{\kappa} \nabla \Psi-i \boldsymbol{A}\right)^{2} \Psi=\Psi\left(|\Psi|^{2}-1\right) \quad \text { in } \Omega \\
-(\operatorname{curl})^{2} \boldsymbol{A}=\left(\frac{\partial \boldsymbol{A}}{\partial t}+\nabla \phi\right)+\frac{i}{2 \kappa}\left(\Psi^{*} \nabla \Psi-\Psi \nabla \Psi^{*}\right)+|\Psi|^{2} \boldsymbol{A} \quad \text { in } \Omega
\end{gathered}
$$

\footnotetext{
1 In fact, there is also a "supercooling field," $H_{s c}$, such that for fields $H_{s c}<H_{0}<H_{c_{1}}$ vortices within the superconductor will remain trapped, even though the global minimum energy solution is that of a completely superconducting sample with no vortices. This will be the subject of another paper.
} 
where $\Omega$ is the region occupied by the superconducting sample, $\Psi$ is the (complex) superconducting order parameter (which can be thought of as a kind of macroscopic wavefunction for the superconducting electrons), and $\boldsymbol{A}$ and $\phi$ are the (real) magnetic vector potential and the electric scalar potential, respectively, which are such that

$$
\boldsymbol{H}=\operatorname{curl} \boldsymbol{A}, \quad \boldsymbol{E}=-\frac{\partial \boldsymbol{A}}{\partial t}-\nabla \phi .
$$

$\boldsymbol{A}$ is unique up to the addition of a gradient; once $\boldsymbol{A}$ is given, $\phi$ is unique up to the addition of a function of $t$. Here $\alpha$ is a positive material constant, and $\kappa$ is the Ginzburg-Landau parameter mentioned in the introduction; we see that $\kappa$ is a ratio of the typical lengthscale for variations of the vector potential (known as the penetration depth, which has been used as the lengthscale in nondimensionalising the equations) to the typical lengthscale for variations in the order parameter (known as the coherence length).

In the steady state, with $\phi=0$, these equations result from minimising the Ginzburg-Landau formulation of the Gibbs free energy [15],

$$
\int\left(-|\Psi|^{2}+\frac{|\Psi|^{4}}{2}+|\boldsymbol{H}|^{2}-2 \boldsymbol{H} \cdot \boldsymbol{H}_{0}+\left|\frac{1}{\kappa} \nabla \Psi-i \boldsymbol{A} \Psi\right|^{2}\right) d V,
$$

where $\boldsymbol{H}_{0}$ is the applied magnetic field. In the time-dependent case they can be obtained as a limiting case of the microscopic BCS equations [2], [16].

We will be concerned only with two-dimensional situations in which the magnetic field is perpendicular to the plane of interest. In this case $\boldsymbol{H}=(0,0, H(x, y, t))$, $\boldsymbol{A}=\left(A_{1}(x, y, t), A_{2}(x, y, t), 0\right)$, and the boundary conditions appropriate to $(1),(2)$ are

$$
\begin{aligned}
\boldsymbol{n} \cdot\left(\frac{1}{\kappa} \nabla-i \boldsymbol{A}\right) \Psi & =0 & \text { on } \partial \Omega, \\
H & =H_{0} & \text { on } \partial \Omega,
\end{aligned}
$$

where $\boldsymbol{n}$ is the unit outward normal to the boundary.

In the units we are using the thermodynamical critical field is given by $H_{c}=1 / \sqrt{2}$, the upper critical field is given by $H_{c_{2}}=\kappa$ (for an infinite sample), and the lower critical field is given by $H_{c_{1}}=(1 / 2 \kappa) \log \kappa$, for large $\kappa$.

Equations (1)-(3) are gauge invariant in the sense that they are invariant under transformations of the type

$$
\boldsymbol{A} \rightarrow \boldsymbol{A}+\nabla \omega, \quad \phi \rightarrow \phi-\frac{\partial \omega}{\partial t}, \quad \Psi \rightarrow \Psi e^{i \kappa \omega} .
$$

We may write the equations in terms of real variables by introducing the new gaugeinvariant potentials

$$
\boldsymbol{Q}=\boldsymbol{A}-\frac{1}{\kappa} \nabla \chi, \quad \Phi=\phi+\frac{1}{\kappa} \frac{\partial \chi}{\partial t},
$$

where $\Psi=f e^{i \chi}$. We then obtain coupled equations for just $f, \boldsymbol{Q}$, and $\phi$ :

$$
\begin{aligned}
& -\frac{\alpha}{\kappa^{2}} \frac{\partial f}{\partial t}+\frac{1}{\kappa^{2}} \nabla^{2} f=f^{3}-f+f|\boldsymbol{Q}|^{2} \quad \text { in } \Omega, \\
& \alpha f^{2} \Phi+\operatorname{div}\left(f^{2} \boldsymbol{Q}\right)=0 \quad \text { in } \Omega \\
& -(\text { curl })^{2} \boldsymbol{Q}=\left(\frac{\partial \boldsymbol{Q}}{\partial t}+\nabla \Phi\right)+f^{2} \boldsymbol{Q} \quad \text { in } \Omega
\end{aligned}
$$


with boundary conditions

$$
\begin{aligned}
\frac{\partial f}{\partial n} & =0 & & \text { on } \partial \Omega, \\
\boldsymbol{Q} \cdot \boldsymbol{n} & =0 & & \text { on } \partial \Omega, \\
H & =H_{0} & & \text { on } \partial \Omega .
\end{aligned}
$$

2.1. Analysis of a halfspace as $\kappa \rightarrow \infty$. We examine the solution of the Ginzburg-Landau equations for the halfspace $x>0$ as the applied external magnetic field is increased. We consider the case of an extreme type II superconductor by setting $\epsilon=1 / \kappa$ and considering the limit as $\epsilon \rightarrow 0$. In one dimension the gauge may be chosen so that $\Psi$ is in fact real and $\boldsymbol{A}=(0, A(x), 0)$. Then $\Psi=f, \boldsymbol{A}=\boldsymbol{Q}$, and the Ginzburg-Landau equations are

$$
\begin{aligned}
\epsilon^{2} f^{\prime \prime} & =f^{3}-f+f Q^{2}, \\
Q^{\prime \prime} & =f^{2} Q \\
H & =Q^{\prime}
\end{aligned}
$$

with boundary conditions

$$
\begin{gathered}
f^{\prime}(0)=0, \quad f^{\prime} \rightarrow 0 \quad \text { as } x \rightarrow \infty, \\
H(0)=H_{0}, \quad Q \rightarrow 0 \quad \text { as } x \rightarrow \infty,
\end{gathered}
$$

where we have assumed that the material at infinity is superconducting. Because of the small parameter multiplying the derivative of $f$ in equation (14), we expect there to be a boundary layer at $x=0$.

Outer solution. We formally expand the outer variables (which we denote by the subscript $o$ ) in powers of $\epsilon$ :

$$
\begin{aligned}
f_{o} & =f_{o}^{(0)}+\epsilon f_{o}^{(1)}+\cdots \\
Q_{o} & =Q_{o}^{(0)}+\epsilon Q_{o}^{(1)}+\cdots \\
H_{o} & =H_{o}^{(0)}+\epsilon H_{o}^{(1)}+\cdots
\end{aligned}
$$

Substituting the expansions (19)-(21) into equations (14), (15) and equating powers of $\epsilon$ yield at leading order

$$
\begin{aligned}
0 & =\left(f_{o}^{(0)}\right)^{3}-f_{o}^{(0)}+f_{o}^{(0)}\left(Q_{o}^{(0)}\right)^{2} \\
\left(Q_{o}^{(0)}\right)^{\prime \prime} & =\left(f_{o}^{(0)}\right)^{2} Q_{o}^{(0)}
\end{aligned}
$$

If $f_{o}^{(0)} \not \equiv 0$, then

$$
\left(f_{o}^{(0)}\right)^{2}=1-\left(Q_{o}^{(0)}\right)^{2}
$$

Substituting this into (23) gives

$$
\left(Q_{o}^{(0)}\right)^{\prime \prime}=Q_{o}^{(0)}-\left(Q_{o}^{(0)}\right)^{3},
$$

with solution

$$
Q_{o}^{(0)}=\frac{2 \sqrt{2} a e^{x}}{1+a^{2} e^{2 x}}
$$


Therefore

$$
H_{o}^{(0)}=\frac{2 \sqrt{2} a e^{x}\left(1-a^{2} e^{2 x}\right)}{\left(1+a^{2} e^{2 x}\right)^{2}} .
$$

This completes the leading-order outer solution, once we have determined the constant $a$. Strictly speaking, because of the boundary layer at $x=0$, the boundary condition (18) should not be applied directly to (26); rather $a$ should be determined by matching with the inner solution. However, when we define the inner variable $X$ by $x=\epsilon X$ and expand in powers of $\epsilon$ as in (19)-(21) in the boundary layer, it is not difficult to show that $Q_{i}$ and $H_{i}$ are constant to leading order (where the subscript $i$ denotes the inner solution), and hence $H_{i}^{(0)}=H_{0}, Q_{i}^{(0)}=b$, say (the boundary layer simply serving to take the derivative of $f$ down to zero). Hence, on matching with the outer solution

$$
\begin{aligned}
H_{0} & =\frac{2 \sqrt{2} a\left(1-a^{2}\right)}{\left(1+a^{2}\right)^{2}}, \\
b & =\frac{2 \sqrt{2} a}{1+a^{2}} .
\end{aligned}
$$

To solve these equations we note that

$$
2\left(H_{o}^{(0)}\right)^{2}=2\left(Q_{o}^{(0)}\right)^{2}-\left(Q_{o}^{(0)}\right)^{4}
$$

and hence

$$
2 b^{2}-b^{4}=2 H_{0}^{2}
$$

with solution

$$
b^{2}=1 \pm \sqrt{1-2 H_{0}^{2}}
$$

The solution for $a$ is now

$$
a^{2}=\frac{4}{b^{2}}-1 \pm \frac{2}{b^{2}} \sqrt{4-2 b^{2}}
$$

However, $a$ must be such that $0 \leq\left(Q_{o}^{(0)}\right)^{2} \leq 1$, giving

$$
a^{2}=\frac{4}{b^{2}}-1+\frac{2}{b^{2}} \sqrt{4-2 b^{2}}, \quad b^{2}=1-\sqrt{1-2 H_{0}^{2}} .
$$

The solution for $f_{i}^{(0)}$ is then

$$
f_{i}^{(0)}=\sqrt{1-b^{2}}=\left(1-2 H_{0}^{2}\right)^{1 / 4} .
$$

Thus we see that the solution exists only for $H_{0} \leq 1 / \sqrt{2}$ (note again that $1 / \sqrt{2}=$ $H_{c}$ in these units). This is the leading-order superheating field obtained by de Gennes $[14]$.

We now perform a local analysis for $H_{0}$ close to $1 / \sqrt{2}$ in order to examine the behaviour of the solution branch at this critical point and to determine to first order correction to the superheating field. 
When $H_{0}=1 / \sqrt{2}$ we find $a=-(1+\sqrt{2})$ and $b=-1$. Hence $Q_{o}^{(0)}(0)=-1$, $f_{o}^{(0)}(0)=0$. In this case a different scaling must be used for the inner solution. As $x \rightarrow 0, f_{o}^{(0)} \sim 2^{1 / 4} x^{1 / 2}$. This, together with (14), indicates the following scaling. We define the inner variable by $x=\epsilon^{2 / 3} X$ and let $f_{i}=\epsilon^{1 / 3} \hat{f}_{i}, Q_{i}=-1+\epsilon^{2 / 3} \hat{Q}_{i}$. Then (14)-(16) become

$$
\begin{aligned}
\hat{f}_{i}^{\prime \prime} & =\hat{f}_{i}^{3}-2 \hat{f}_{i} \hat{Q}_{i}+\epsilon^{2 / 3} \hat{f}_{i} \hat{Q}_{i}^{2} \\
\hat{Q}_{i}^{\prime \prime} & =-\epsilon^{4 / 3} \hat{f}_{i}^{2}+\epsilon^{2} \hat{f}_{i}^{2} \hat{Q}_{i} \\
H_{i} & =\hat{Q}_{i}^{\prime} .
\end{aligned}
$$

We expand $\hat{f}_{i}, \hat{Q}_{i}$, and $H_{i}$ in powers of $\epsilon^{2 / 3}$ :

$$
\begin{aligned}
\hat{f}_{i} & =\hat{f}_{i}^{(0)}+\epsilon^{2 / 3} \hat{f}_{i}^{(1)}+\epsilon^{4 / 3} \hat{f}_{i}^{(2)}+\cdots, \\
\hat{Q}_{i} & =\hat{Q}_{i}^{(0)}+\epsilon^{2 / 3} \hat{Q}_{i}^{(1)}+\epsilon^{4 / 3} \hat{Q}_{i}^{(2)}+\cdots, \\
H_{i} & =H_{i}^{(0)}+\epsilon^{2 / 3} H_{i}^{(1)}+\epsilon^{4 / 3} H_{i}^{(2)}+\cdots
\end{aligned}
$$

Substituting these expansions into equations (30)-(32) and equating powers of $\epsilon$ yield at leading order:

$$
\begin{aligned}
\left(\hat{f}_{i}^{(0)}\right)^{\prime \prime} & =\left(\hat{f}_{i}^{(0)}\right)^{3}-2 \hat{f}_{i}^{(0)} \hat{Q}_{i}^{(0)} \\
\left(\hat{Q}_{i}^{(0)}\right)^{\prime \prime} & =0 \\
H_{i}^{(0)} & =\left(\hat{Q}_{i}^{(0)}\right)^{\prime} .
\end{aligned}
$$

Hence

$$
\hat{Q}_{i}^{(0)}=\frac{X+C}{\sqrt{2}}
$$

The equation for $\hat{f}_{i}^{(0)}$ is now the equation for the second Painlevé transcendent and cannot be solved in terms of elementary functions. To match with the outer solution we require the solution that has the asymptotic form

$$
\hat{f}_{i}^{(0)} \sim 2^{1 / 4} X^{1 / 2} \text { as } X \rightarrow \infty .
$$

Let us return for the moment to the outer solution. Because of the form of the inner solution, we expect the outer expansions also to be in powers of $\epsilon^{2 / 3}$ :

$$
\begin{aligned}
f_{o} & =f_{o}^{(0)}+\epsilon^{2 / 3} f_{o}^{(1)}+\epsilon^{4 / 3} f_{o}^{(2)}+\cdots, \\
Q_{o} & =Q_{o}^{(0)}+\epsilon^{2 / 3} Q_{o}^{(1)}+\epsilon^{4 / 3} Q_{o}^{(2)}+\cdots, \\
H_{o} & =H_{o}^{(0)}+\epsilon^{2 / 3} H_{o}^{(1)}+\epsilon^{4 / 3} H_{o}^{(2)}+\cdots .
\end{aligned}
$$

Substituting the expansions into (14) and equating coefficients of $\epsilon^{2 / 3}$ yield, using (33),

$$
f_{o}^{(1)}=-\frac{Q_{o}^{(0)} Q_{o}^{(1)}}{f_{o}^{(0)}} .
$$

To solve for $Q_{o}^{(1)}$ we first note that equations (14)-(15) have a first integral given by

$$
\epsilon^{2}\left(f^{\prime}\right)^{2}+\left(Q^{\prime}\right)^{2}=\frac{\left(1-f^{2}\right)^{2}}{2}+f^{2} Q^{2}
$$


where we have applied the boundary conditions at infinity to eliminate the constant of integration. We equate coefficients of $\epsilon^{2 / 3}$ in this equation to give, using (33) and $(36)$,

$$
\left(Q_{o}^{(0)}\right)^{\prime}\left(Q_{o}^{(1)}\right)^{\prime}=\left(Q_{o}^{(0)}-\left(Q_{o}^{(0)}\right)^{3}\right) Q_{o}^{(1)}
$$

with solution

$$
Q_{o}^{(1)}=D\left(Q_{o}^{(0)}\right)^{\prime}=\frac{2 \sqrt{2} D a e^{x}\left(1-a^{2} e^{2 x}\right)}{\left(1+a^{2} e^{2 x}\right)^{2}},
$$

where $D$ is arbitrary at this point. This gives

$$
\begin{aligned}
H_{o}^{(1)} & =D\left(Q_{o}^{(0)}-\left(Q_{o}^{(0)}\right)^{3}\right) \\
& =\frac{D a e^{x}\left(1-6 a^{2} e^{2 x}+a^{4} e^{4 x}\right)}{\left(1+a^{2} e^{2 x}\right)^{3}} .
\end{aligned}
$$

We proceed to one more term in the outer solution. Equating coefficients of $\epsilon^{4 / 3}$ in (14) and (37) and using (33), (36), and (38) yield

$$
\begin{aligned}
f_{o}^{(2)} & =-\frac{Q_{o}^{(0)} Q_{o}^{(2)}}{f_{o}^{(0)}}-\frac{D^{2}\left(\left(Q_{o}^{(0)}\right)^{\prime}\right)^{2}}{2\left(f_{o}^{(0)}\right)^{3}} \\
\left(Q_{o}^{(2)}\right)^{\prime}+\frac{\left(\left(Q_{o}^{(0)}\right)^{3}-Q_{o}^{(0)}\right) Q_{o}^{(2)}}{\left(Q_{o}^{(0)}\right)^{\prime}} & =\frac{D^{2}\left(Q_{o}^{(0)}\right)^{4}\left(\left(Q_{o}^{(0)}\right)^{2}-3\right)}{4\left(Q_{o}^{(0)}\right)^{\prime}}
\end{aligned}
$$

with solution

$$
\begin{aligned}
Q_{o}^{(2)} & =\frac{2 \sqrt{2} a D^{2} e^{x}\left(1-3 a^{2} e^{2 x}\right)}{\left(1+a^{2} e^{2 x}\right)^{3}}+\frac{E a e^{x}\left(a^{2} e^{2 x}-1\right)}{\left(1+a^{2} e^{2 x}\right)^{2}} \\
H_{o}^{(2)} & =\frac{2 \sqrt{2} a D^{2} e^{x}\left(1-14 a^{2} e^{2 x}+9 a^{4} e^{4 x}\right)}{\left(1+a^{2} e^{2 x}\right)^{4}}-\frac{E a e^{x}\left(1-6 a^{2} e^{2 x}+a^{4} e^{4 x}\right)}{\left(1+a^{2} e^{2 x}\right)^{3}}
\end{aligned}
$$

where, again, $E$ is arbitrary at this point.

When we match with the inner solution, we will need to know the limiting forms of the outer solution as $x \rightarrow 0$. We have

$$
\begin{aligned}
& Q_{o}^{(0)} \sim-1+\frac{x}{\sqrt{2}}-\frac{x^{3}}{3 \sqrt{2}}+\cdots, \\
& Q_{o}^{(1)} \sim \frac{D}{\sqrt{2}}-\frac{D x^{2}}{\sqrt{2}}+\cdots, \\
& Q_{o}^{(2)} \sim \frac{D^{2}}{2 \sqrt{2}}-\frac{E}{4}-\frac{D^{2} x}{\sqrt{2}}+\cdots, \\
& H_{o}^{(0)} \sim \frac{1}{\sqrt{2}}-\frac{x^{2}}{\sqrt{2}}+\frac{x^{3}}{2}+\cdots, \\
& H_{o}^{(1)} \sim-\sqrt{2} D x+\frac{3 D x^{2}}{2}+\cdots, \\
& H_{o}^{(2)} \sim-\frac{D^{2}}{\sqrt{2}}+\cdots, \\
& f_{o}^{(0)} \sim 2^{1 / 4} x^{1 / 2}-\frac{x^{3 / 2}}{2^{1 / 4} .4}+\cdots,
\end{aligned}
$$




$$
\begin{aligned}
f_{o}^{(1)} & \sim \frac{D}{2^{3 / 4} \sqrt{x}}-\frac{3 D \sqrt{x}}{2^{1 / 4} .8}+\cdots \\
f_{o}^{(2)} & \sim-\frac{D^{2}}{2^{3 / 4} .4 x^{3 / 2}}+\cdots
\end{aligned}
$$

Let us now turn again to the inner solution. We have

$$
Q_{i} \sim-1+\epsilon^{2 / 3} \frac{X+C}{\sqrt{2}} .
$$

Hence

$$
\begin{aligned}
& (1 \mathrm{to})(2 \mathrm{ti})=-1+\frac{x}{\sqrt{2}}, \\
& (2 \mathrm{to})(2 \mathrm{ti})=-1+\frac{x}{\sqrt{2}}+\epsilon^{2 / 3} \frac{C}{\sqrt{2}} .
\end{aligned}
$$

We have from the outer expansion

$$
(2 \mathrm{ti})(2 \mathrm{to})=-1+\epsilon^{2 / 3} \frac{X}{\sqrt{2}}+\epsilon^{2 / 3} \frac{D}{\sqrt{2}} .
$$

Hence $C=D$.

We now turn our attention to $H_{i}$. We have

$$
\left(H_{i}^{(1)}\right)^{\prime}=0 .
$$

Matching with the outer solution implies $H_{i}^{(1)}=0$. Equating powers of $\epsilon$ at the next order in (32) yields

$$
\left(H_{i}^{(2)}\right)^{\prime}=-\left(f_{i}^{(0)}\right)^{2}
$$

Hence

$$
H_{i}^{(2)}=H_{0}^{(2)}-\int_{0}^{y}\left(f_{i}^{(0)}\right)^{2} d y
$$

where $H_{0}=1 / \sqrt{2}+\epsilon^{4 / 3} H_{0}^{(2)}$. Matching this term with the outer solution will give the constant $D$ in terms of $H_{0}^{(2)}$. Writing $H_{i}$ in terms of the outer variable $x$ and expanding give

$$
\begin{aligned}
& (1 \mathrm{to})(3 \mathrm{ti})=\frac{1}{\sqrt{2}}-\frac{x^{2}}{\sqrt{2}} \\
& (2 \mathrm{to})(3 \mathrm{ti})=\frac{1}{\sqrt{2}}-\frac{x^{2}}{\sqrt{2}}-\sqrt{2} \epsilon^{2 / 3} D x \\
& (3 \mathrm{to})(3 \mathrm{ti})=\frac{1}{\sqrt{2}}-\frac{x^{2}}{\sqrt{2}}-\sqrt{2} \epsilon^{2 / 3} D x+\epsilon^{4 / 3} H_{0}^{(2)}-\epsilon^{4 / 3} \beta
\end{aligned}
$$

where

$$
\beta=\int_{0}^{\infty}\left[\left(f_{i}^{(0)}\right)^{2}-\sqrt{2}(X+D)\right] d X
$$




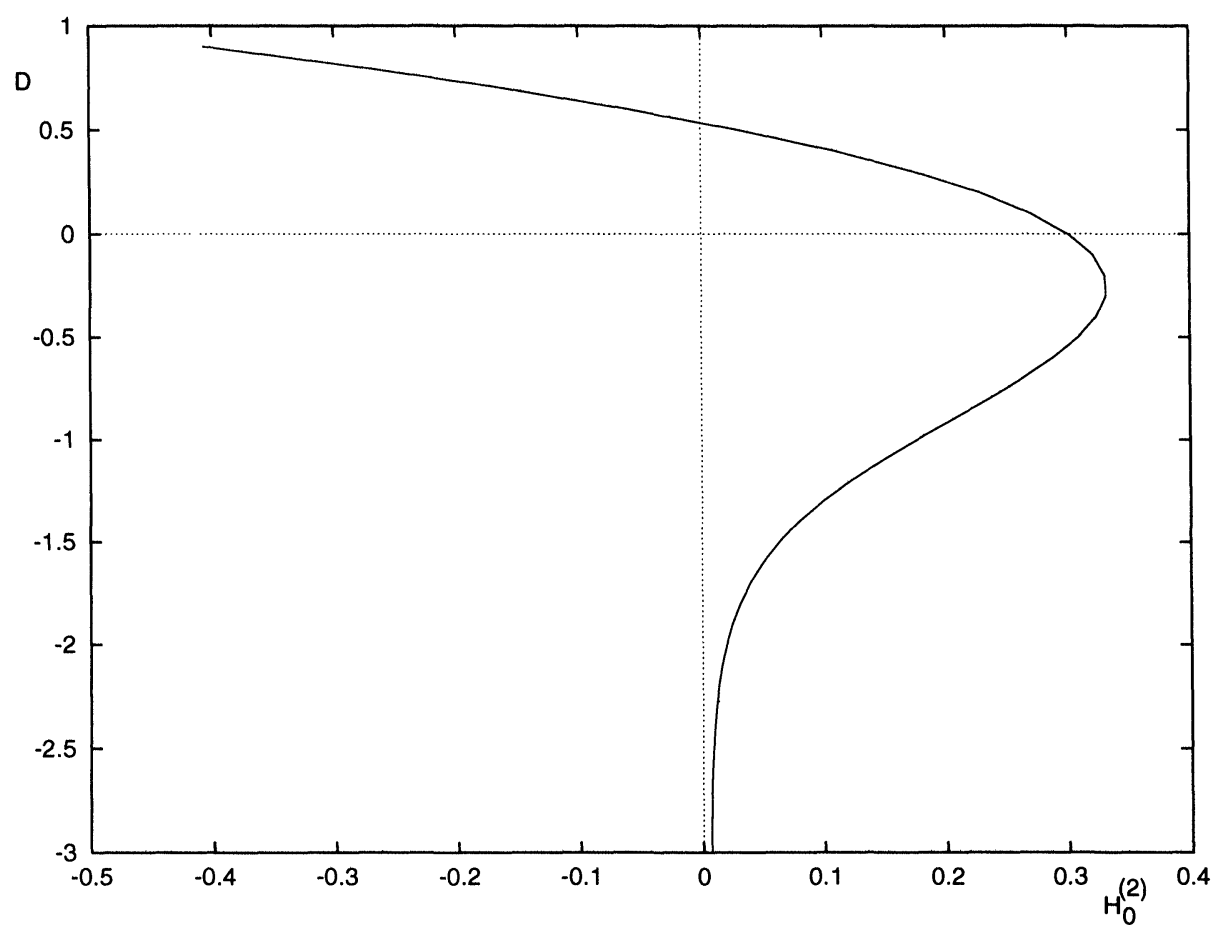

FIG. 2. $D$ as a function of $H_{0}^{(2)}$.

From the outer solution we find

$$
(3 \text { ti })(3 \text { to })=\frac{1}{\sqrt{2}}-\epsilon^{4 / 3} \frac{X^{2}}{\sqrt{2}}-\sqrt{2} \epsilon^{4 / 3} D X-\epsilon^{4 / 3} \frac{D^{2}}{\sqrt{2}} .
$$

Hence

$$
H_{0}^{(2)}=\beta-\frac{D^{2}}{\sqrt{2}} .
$$

Figure 2 shows a plot of $D$ against $H_{0}^{(2)}$. We see that there is a folding over of the response diagram as the solution branch turns back on itself. The value of $H_{0}$ at the nose gives the limiting field for the existence of this solution branch. Above this field the solution must switch to some other solution branch (which will correspond to changing into the mixed state).

This shows the limit of the existence of the superconducting solution. However, to determine the superheating field we need to examine the stability of the superconducting solution.

3. Linear stability in one dimension. We consider the linear stability of the solution. In one dimension, the time-dependent Ginzburg-Landau equations are

$$
\begin{aligned}
-\alpha \epsilon^{2} \frac{\partial f}{\partial t}+\epsilon^{2} \frac{\partial^{2} f}{\partial x^{2}} & =f^{3}-f+f Q^{2} \\
-\frac{\partial Q}{\partial t}+\frac{\partial^{2} Q}{\partial x^{2}} & =f^{2} Q \\
H & =\frac{\partial Q}{\partial x}
\end{aligned}
$$


We denote the previously found steady-state solution by $\left(f_{0}, Q_{0}\right)$ and make a small perturbation about it by setting

$$
\begin{aligned}
f & =f_{0}+\delta e^{\sigma t} f_{1}(x), \\
Q & =Q_{0}+\delta e^{\sigma t} Q_{1}(x), \\
H & =H_{0}+\delta e^{\sigma t} H_{1}(x), \quad 0<\delta \ll 1 .
\end{aligned}
$$

Substituting these expressions into (48)-(50) and linearising in $\delta$ (to give the leadingorder behaviour of an asymptotic expansion in powers of $\delta$ ) yield

$$
\begin{aligned}
-\alpha \epsilon^{2} \sigma f_{1}+\epsilon^{2} \frac{\partial^{2} f_{1}}{\partial x^{2}} & =3 f_{0}^{2} f_{1}-f_{1}+f_{1} Q_{0}^{2}+2 f_{0} Q_{0} Q_{1} \\
-\sigma Q_{1}+\frac{\partial^{2} Q_{1}}{\partial x^{2}} & =f_{0}^{2} Q_{1}+2 f_{0} f_{1} Q_{0} \\
H_{1} & =\frac{\partial Q_{1}}{\partial x}
\end{aligned}
$$

with boundary conditions

$$
\begin{aligned}
\frac{\partial f_{1}}{\partial x}(0) & =0, & & H_{1}(0)=0, \\
\frac{\partial f_{1}}{\partial x} & \rightarrow 0, & H_{1} \rightarrow 0 & \text { as } x \rightarrow \infty .
\end{aligned}
$$

We now consider an asymptotic expansion in powers of $\epsilon$ as before:

$$
\begin{aligned}
Q_{o, 1} & =Q_{o, 1}^{(0)}+\epsilon Q_{o, 1}^{(1)}+\cdots, \\
f_{o, 1} & =f_{o, 1}^{(0)}+\epsilon f_{o, 1}^{(1)}+\cdots, \\
H_{o, 1} & =H_{o, 1}^{(0)}+\epsilon H_{o, 1}^{(1)}+\cdots .
\end{aligned}
$$

Note that since we are expanding in powers of $\epsilon$ after linearising in $\delta$, we are assuming that $\delta \ll \epsilon$. As before, we will need to consider both the outer solution and the inner boundary layer. At leading order in the outer region we have, using (33),

$$
\begin{aligned}
f_{o, 1}^{(0)} & =-\frac{Q_{o, 0}^{(0)} Q_{o, 1}^{(0)}}{f_{o, 0}^{(0)}} \\
\frac{\partial^{2} Q_{o, 1}^{(0)}}{\partial x^{2}} & =\left(\sigma^{(0)}+1-3\left(Q_{o, 0}^{(0)}\right)^{2}\right) Q_{o, 1}^{(0)} .
\end{aligned}
$$

We also have that

$$
\frac{\partial f_{o, 1}^{(0)}}{\partial x} \rightarrow 0, \frac{\partial Q_{o, 1}^{(0)}}{\partial x} \rightarrow 0, \text { as } x \rightarrow \infty .
$$

To obtain a boundary condition at $x=0$ we must consider the inner solution.

As before we define the inner variable $X$ by $x=\epsilon X$. Equations (51)-(53) become

$$
\begin{aligned}
-\alpha \epsilon^{2} \sigma f_{i, 1}+\frac{\partial^{2} f_{i, 1}}{\partial X^{2}} & =3 f_{i, 0}^{2} f_{i, 1}-f_{i, 1}+f_{i, 1} Q_{i, 0}^{2}+2 f_{i, 0} Q_{i, 0} Q_{i, 1} \\
-\epsilon \sigma Q_{i, 1}+\frac{\partial H_{i, 1}}{\partial X} & =\epsilon\left(f_{i, 0}^{2} Q_{i, 1}+2 f_{i, 0} f_{i, 1} Q_{i, 0}\right) \\
\epsilon H_{i, 1} & =\frac{\partial Q_{i, 1}}{\partial X}
\end{aligned}
$$


with boundary conditions

$$
\begin{aligned}
\frac{\partial f_{i, 1}}{\partial y}(0) & =0 \\
H_{i, 1}(0) & =0 .
\end{aligned}
$$

We find $\partial H_{i, 1}^{(0)} / \partial X=0$ and hence $H_{i, 1}^{(0)}=0$. Matching with the outer solution implies $\partial Q_{o, 1}^{(0)} / \partial x(0)=0$. Now, multiplying $(55)$ by $Q_{o, 1}^{(0)}$ and integrating over $[0, \infty)$ yield, after integration by parts,

$$
\sigma^{(0)} \int_{0}^{\infty}\left(Q_{o, 1}^{(0)}\right)^{2} d x=-\int_{0}^{\infty}\left(\frac{\partial Q_{o, 1}^{(0)}}{\partial x}\right)^{2} d x+\int_{0}^{\infty}\left(3\left(Q_{o, 0}^{(0)}\right)^{2}-1\right)\left(Q_{o, 1}^{(0)}\right)^{2} d x
$$

Now, for $H_{0}$ small, $\left(Q_{o, 1}^{(0)}\right)^{2}<1 / 3$, and hence $\sigma^{(0)}<0$. Hence the solution is linearly stable for small $H_{0}$. We expect each of the eigenvalues $\sigma^{(0)}<0$ to depend continuously on $H_{0}$. When an eigenvalue passes through zero, we know that the only solution of (55) is $Q_{o, 0}^{(1)} \propto d Q_{o, 0}^{(0)} / d x$. In order for this to satisfy the boundary condition at $x=0$ we require $d^{2} Q_{o, 0}^{(0)} / d x^{2}(0)=0$. This is only true when $H_{0}=1 / \sqrt{2}$. Thus we expect all eigenvalues $\sigma^{(0)}$ to be negative for $H_{0}<1 / \sqrt{2}$. When $H_{0}=1 / \sqrt{2}$ the largest eigenvalue will be zero to leading order. Hence we need to proceed to higher orders in the expansion to determine the stability of the solution in the neighbourhood of $H_{0}=1 / \sqrt{2}$.

3.1. $H_{0}$ close to $1 / \sqrt{2}$. We have the leading-order behaviour of the perturbation

$$
\begin{aligned}
Q_{o, 1}^{(0)} & =\frac{d Q_{o, 0}^{(0)}}{d x}, \\
f_{o, 1}^{(0)} & =-\frac{Q_{o, 0}^{(0)}}{f_{o, 0}^{(0)}} \frac{d Q_{o, 0}^{(0)}}{d x} .
\end{aligned}
$$

As in the steady state, we assume an expansion in powers of $\epsilon^{2 / 3}$ :

$$
\begin{aligned}
Q_{o, 1} & =Q_{o, 1}^{(0)}+\epsilon^{2 / 3} Q_{o, 1}^{(1)}+\cdots \\
f_{o, 1} & =f_{o, 1}^{(0)}+\epsilon^{2 / 3} f_{o, 1}^{(1)}+\cdots \\
H_{o, 1} & =H_{o, 1}^{(0)}+\epsilon^{2 / 3} H_{o, 1}^{(1)}+\cdots
\end{aligned}
$$

Substituting these expansions into (51)-(53), equating coefficients of $\epsilon^{2 / 3}$, and using $(33),(36),(61)$, and (62) yield

$$
\begin{aligned}
f_{o, 1}^{(1)} & =-\frac{Q_{o, 0}^{(0)} Q_{o, 1}^{(1)}}{f_{o, 0}^{(0)}}-\frac{D}{\left(f_{o, 0}^{(0)}\right)^{3}}\left(\frac{d Q_{o, 0}^{(0)}}{d x}\right)^{2} \\
\frac{\partial^{2} Q_{o, 1}^{(1)}}{\partial x^{2}}+\left(3\left(Q_{o, 0}^{(0)}\right)^{2}-1\right) Q_{o, 1}^{(1)} & =\sigma^{(1)} \frac{d Q_{o, 0}^{(0)}}{d x}-6 D Q_{o, 0}^{(0)}\left(\frac{d Q_{o, 0}^{(0)}}{d x}\right)^{2}
\end{aligned}
$$

where $D$ is as before. Now $d Q_{o, 0}^{(0)} / d x$ is a solution of the homogeneous version of (64). Note though that we will find when we match with the inner solution that the 
boundary conditions on $Q_{o, 1}^{(1)}$ are not homogeneous. We multiply by $d Q_{o, 0}^{(0)} / d x$ and integrate to give

$$
\begin{aligned}
{\left[\frac{d Q_{o, 0}^{(0)}}{d x} \frac{\partial Q_{o, 1}^{(1)}}{\partial x}-\frac{d^{2} Q_{o, 0}^{(0)}}{d x^{2}} Q_{o, 1}^{(1)}\right]_{0}^{\infty}=} & \sigma^{(1)} \int_{0}^{\infty}\left(\frac{d Q_{o, 0}^{(0)}}{d x}\right)^{2} d x \\
& -6 D \int_{-1}^{0} Q_{o, 0}^{(0)}\left(\left(Q_{o, 0}^{(0)}\right)^{2}-\frac{\left(Q_{o, 0}^{(0)}\right)^{4}}{2}\right) d\left(Q_{o, 0}^{(0)}\right) \\
= & \sigma^{(1)} \int_{0}^{\infty}\left(\frac{d Q_{o, 0}^{(0)}}{d x}\right)^{2} d x+D
\end{aligned}
$$

Since $d Q_{o, 0}^{(0)} / d x, d^{2} Q_{o, 0}^{(0)} / d x^{2} \rightarrow 0$ as $x \rightarrow \infty$, and $d Q_{o, 0}^{(0)} / d x(0)=1 / \sqrt{2}, d^{2} Q_{o, 0}^{(0)} / d x^{2}(0)=$ 0 , we have

$$
\sigma^{(1)} \int_{0}^{\infty}\left(\frac{d Q_{o, 0}^{(0)}}{d x}\right)^{2} d x=-D-\frac{1}{\sqrt{2}} \frac{\partial Q_{o, 1}^{(1)}}{\partial x}(0)
$$

In order to determine $\partial Q_{o, 1}^{(1)} / \partial x(0)$ we turn our attention to the inner solution. As in the steady state we define the inner variable $X$ by $x=\epsilon^{2 / 3} X$ and new variables $\hat{f}_{i, 0}$ and $\hat{Q}_{i, 0}$ by

$$
\begin{aligned}
f_{i, 0} & =\epsilon^{1 / 3} \hat{f}_{i, 0}, \\
Q_{i, 0} & =-1+\epsilon^{2 / 3} \hat{Q}_{i, 0} .
\end{aligned}
$$

Equations (51)-(53) then become

$$
\begin{aligned}
-\epsilon^{2} \sigma f_{i, 1}+\epsilon^{2 / 3} \frac{\partial^{2} f_{i, 1}}{\partial X^{2}}= & 3 \epsilon^{2 / 3}\left(\hat{f}_{i, 0}\right)^{2} f_{i, 1}-2 \epsilon^{2 / 3} f_{i, 1} \hat{Q}_{i, 0}+\epsilon^{4 / 3} f_{i, 1}\left(\hat{Q}_{i, 0}\right)^{2} \\
& -2 \epsilon^{1 / 3} \hat{f}_{i, 0} Q_{i, 1}+2 \epsilon \hat{f}_{i, 0} \hat{Q}_{i, 0} Q_{i, 1} \\
-\epsilon^{4 / 3} \sigma Q_{i, 1}+\frac{\partial^{2} Q_{i, 1}}{\partial X^{2}}= & \epsilon^{2}\left(\hat{f}_{i, 0}\right)^{2} Q_{i, 1}-2 \epsilon^{5 / 3} f_{i, 1} \hat{f}_{i, 0}+2 \epsilon^{7 / 3} f_{i, 1} \hat{Q}_{i, 0} \hat{f}_{i, 0} \\
H_{i, 1}= & \frac{\partial \hat{Q}_{i, 0}}{\partial X}
\end{aligned}
$$

We see that the correct scaling for $f_{i, 1}$ is $f_{i, 1}=\epsilon^{-1 / 3} \hat{f}_{i, 1}$. The leading-order behaviour of $\hat{f}_{i, 1}$ is then given by

$$
\frac{\partial^{2} \hat{f}_{i, 1}^{(0)}}{\partial X^{2}}=3\left(\hat{f}_{i, 0}^{(0)}\right)^{2} \hat{f}_{i, 1}^{(0)}-\sqrt{2}(X+D) \hat{f}_{i, 1}^{(0)}-\sqrt{2} \hat{f}_{i, 0}^{(0)}
$$

with boundary conditions

$$
\begin{aligned}
\frac{\partial \hat{f}_{i, 1}^{(0)}}{\partial X}(0) & =0 \\
\hat{f}_{i, 1}^{(0)} & \sim \frac{1}{2^{3 / 4} \sqrt{X}} \text { as } X \rightarrow \infty
\end{aligned}
$$


The solution of this equation is

$$
\hat{f}_{i, 1}^{(0)}=\frac{\partial \hat{f}_{i, 0}^{(0)}}{\partial D} .
$$

We see that the leading-order behaviour of $H_{i, 1}$ is given by $H_{i, 1} \sim \epsilon^{4 / 3} H_{i, 1}^{(2)}$, where

$$
H_{i, 1}^{(2)}=-\int_{0}^{X} \frac{\partial\left[\left(\hat{f}_{i, 0}^{(0)}\right)^{2}\right]}{\partial D} d X
$$

Now

$$
\begin{aligned}
(2 \mathrm{ti})(2 \mathrm{to})=-\epsilon^{2 / 3} \sqrt{2} X+\epsilon^{2 / 3} \frac{\partial Q_{o, 1}^{(1)}}{\partial x}(0) \\
(2 \mathrm{to})(2 \mathrm{ti})=-\sqrt{2} x-\epsilon^{2 / 3} \int_{0}^{\infty}\left\{\frac{\partial\left[\left(\hat{f}_{i, 0}^{(0)}\right)^{2}\right]}{\partial D}-\sqrt{2}\right\} d X \\
=-\epsilon^{2 / 3} \sqrt{2} X-\epsilon^{2 / 3} \int_{0}^{\infty} \frac{\partial}{\partial D}\left[\left(\hat{f}_{i, 0}^{(0)}\right)^{2}-\sqrt{2}(X+D)\right] d X \\
=-\epsilon^{2 / 3} \sqrt{2} X-\epsilon^{2 / 3} \frac{\partial}{\partial D} \int_{0}^{\infty}\left[\left(\hat{f}_{i, 0}^{(0)}\right)^{2}-\sqrt{2}(X+D)\right] d X \\
=-\epsilon^{2 / 3} \sqrt{2} X-\epsilon^{2 / 3} \frac{\partial \beta}{\partial D} .
\end{aligned}
$$

Hence, by matching

$$
\frac{\partial Q_{o, 1}^{(1)}}{\partial x}(0)=-\frac{\partial \beta}{\partial D}
$$

Hence

$$
\begin{aligned}
\sigma^{(1)} \int_{0}^{\infty}\left(\frac{d Q_{o, 0}^{(0)}}{d x}\right)^{2} d x & =\frac{1}{\sqrt{2}} \frac{\partial \beta}{\partial D}-D \\
& =\frac{1}{\sqrt{2}} \frac{\partial}{\partial D}\left[\beta-\frac{D^{2}}{\sqrt{2}}\right] \\
& =\frac{1}{\sqrt{2}} \frac{\partial H_{0}^{(2)}}{\partial D} .
\end{aligned}
$$

Hence $\sigma^{(1)}<0$ if and only if $\partial H_{0}^{(2)} / \partial D<0$. Thus we see that the stability of the solution branch switches as we pass through the nose in Fig. 2, with the upper branch being stable, and the lower branch unstable, as shown in Fig. 3. Thus in one dimension the superheating field is given by the field at the nose in Fig. 2 and is approximately $1 / \sqrt{2}+0.33 / \kappa^{4 / 3}$.

4. Linear stability in two dimensions. In this section we consider the stability of the solution with respect to two-dimensional perturbations. When we wrote the one-dimensional equations we were implicitly choosing a gauge by writing the 


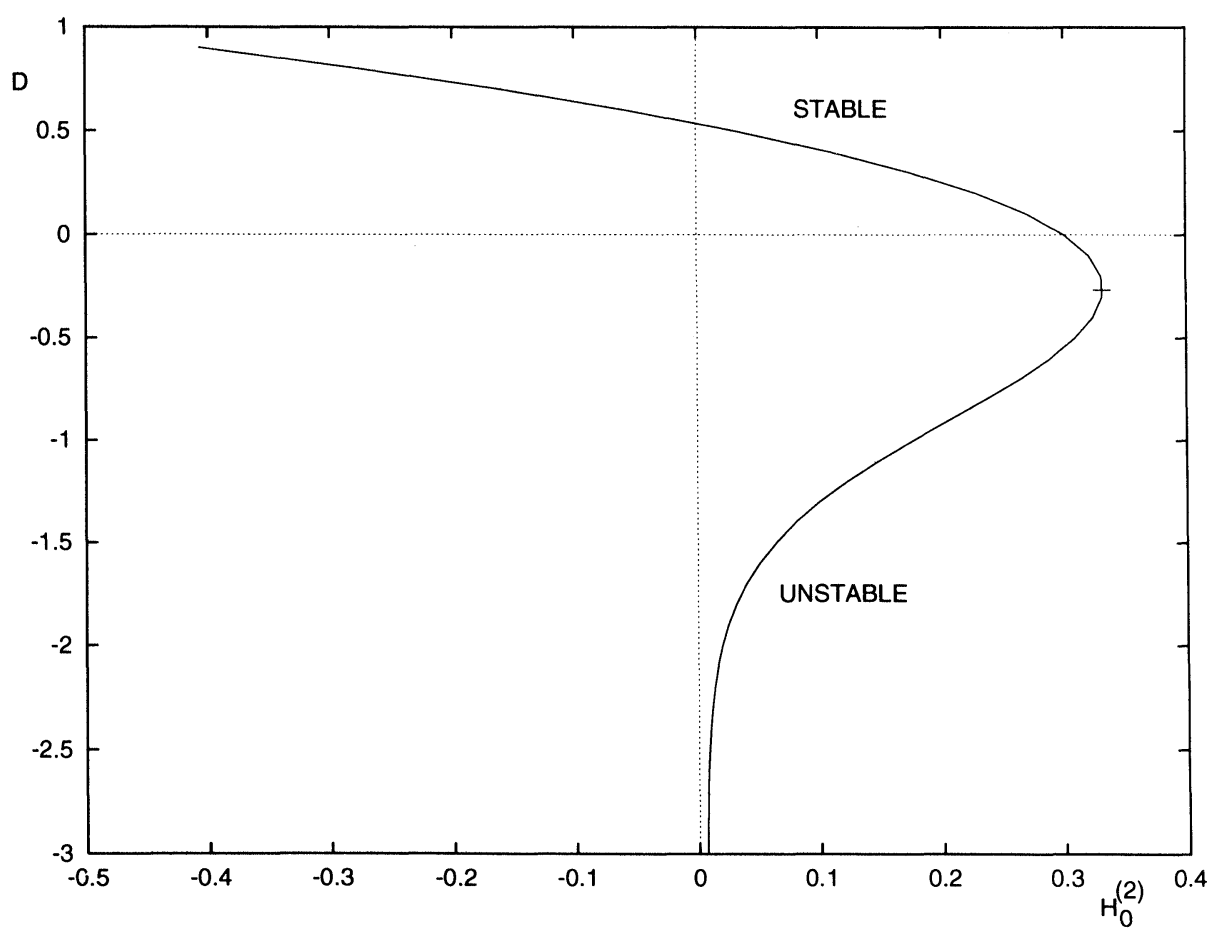

FIG. 3. $D$ as a function of $H_{0}^{(2)}$, showing the stability of the solution branches.

order parameter real and taking the vector potential to be directed in the $y$-direction. Since we are going to perturb about the one-dimensional solution that we obtained, we should choose a compatible gauge even though we are now in two dimensions. Hence we again take the vector potential to be directed in the $y$-direction, so that $\boldsymbol{A}=(0, A(x, y), 0)$ and $\boldsymbol{H}=(0,0, H)$. We also take $A$ and $\phi$ to tend to zero as $x \rightarrow \infty$. The Ginzburg-Landau equations (1), (2) then become

(65) $\epsilon^{2}\left(\frac{\partial^{2} \Psi}{\partial x^{2}}+\frac{\partial^{2} \Psi}{\partial y^{2}}\right)=\alpha \epsilon^{2} \frac{\partial \Psi}{\partial t}+\alpha \epsilon i \Psi \phi-2 i \epsilon A \frac{\partial \Psi}{\partial y}-i \epsilon \Psi \frac{\partial A}{\partial y}$

$$
\begin{aligned}
-\frac{\partial^{2} A}{\partial x \partial y} & =\frac{\partial \phi}{\partial x}+\frac{i \epsilon}{2}\left(\Psi^{*} \frac{\partial \Psi}{\partial x}-\Psi \frac{\partial \Psi^{*}}{\partial x}\right) \\
\frac{\partial^{2} A}{\partial x^{2}} & =\frac{\partial \phi}{\partial y}+\frac{\partial A}{\partial t}+\frac{i \epsilon}{2}\left(\Psi^{*} \frac{\partial \Psi}{\partial y}-\Psi \frac{\partial \Psi^{*}}{\partial y}\right)+|\psi|^{2} A \\
H & =\frac{\partial A}{\partial x}
\end{aligned}
$$

with boundary conditions

$$
\begin{aligned}
\frac{\partial \Psi}{\partial x}(0, y) & =0 \\
H(0, y) & =H_{0} \\
A, \quad H, \quad \phi, \quad \frac{\partial \Psi}{\partial x} & \rightarrow 0 \text { as } x \rightarrow \infty
\end{aligned}
$$


We consider a small two-dimensional perturbation of the one-dimensional solution found previously. Now, as we have seen, there are two lengthscales and two timescales in the problem. We consider first perturbations varying on the longer lengthscale (i.e., that of the penetration depth) by setting

$$
\begin{aligned}
\Psi & =f(x)+\delta e^{\sigma t} \Psi_{1}(x, y), \\
A & =Q(x)+\delta e^{\sigma t} A_{1}(x, y), \\
\phi & =\delta e^{\sigma t} \phi_{1}(x, y) .
\end{aligned}
$$

Substituting these expressions into (65)-(67) and linearising in $\delta$ yield

$$
\begin{aligned}
\epsilon^{2} \frac{\partial^{2} \Psi_{1}}{\partial x^{2}}+\epsilon^{2} \frac{\partial^{2} \Psi_{1}}{\partial y^{2}}= & \alpha \epsilon^{2} \frac{\partial \Psi_{1}}{\partial t}+\alpha \epsilon i f \phi_{1}-2 i \epsilon Q \frac{\partial \Psi_{1}}{\partial y}-i \epsilon f \frac{\partial A_{1}}{\partial y} \\
& +2 A_{1} Q f+Q^{2} \Psi_{1}+f^{2}\left(2 \Psi_{1}+\Psi_{1}^{*}\right)-\Psi_{1} \\
-\frac{\partial^{2} A_{1}}{\partial x \partial y}= & \frac{\partial \phi_{1}}{\partial x}+\frac{i \epsilon}{2}\left(f \frac{\partial \Psi_{1}}{\partial x}-f \frac{\partial \Psi_{1}^{*}}{\partial x}+\Psi_{1}^{*} \frac{d f}{d x}-\Psi_{1} \frac{d f}{d x}\right) \\
\frac{\partial^{2} A_{1}}{\partial x^{2}}= & \frac{\partial \phi_{1}}{\partial y}+\frac{\partial A_{1}}{\partial t}+\frac{i \epsilon}{2}\left(f \frac{\partial \Psi_{1}}{\partial y}-f \frac{\partial \Psi_{1}^{*}}{\partial y}\right) \\
& +f^{2} A_{1}+f Q\left(\psi_{1}+\Psi_{1}^{*}\right) .
\end{aligned}
$$

Again we consider inner and outer solutions. Expanding in powers of $\epsilon$ yields the leading-order outer problem (dropping the 1's)

(79)- $\frac{\partial^{2} A_{o}^{(0)}}{\partial x \partial y}=\frac{\partial \phi_{o}^{(0)}}{\partial x}$,

(80) $\frac{\partial^{2} A_{o}^{(0)}}{\partial x^{2}}=\frac{\partial \phi_{o}^{(0)}}{\partial y}+\sigma^{(0)} A_{o}^{(0)}+\left(f_{o}^{(0)}\right)^{2} A_{o}^{(0)}+f_{o}^{(0)} Q_{o}^{(0)}\left(\Psi_{o}^{(0) *}+\Psi_{o}^{(0)}\right)$.

We let $\Psi_{o}^{(0)}=u+i v$, where $u$ and $v$ are real. Then we find that $v$ is arbitrary,

$$
\begin{aligned}
u & =-\frac{Q_{o}^{(0)} A_{o}^{(0)}}{f_{o}^{(0)}} \\
\phi_{o}^{(0)} & =-\frac{\partial A_{o}^{(0)}}{\partial y} \\
\frac{\partial^{2} A_{o}^{(0)}}{\partial x^{2}}+\frac{\partial^{2} A_{o}^{(0)}}{\partial y^{2}} & =\sigma^{(0)} A_{o}^{(0)}+\left(1-3\left(Q_{o}^{(0)}\right)^{2}\right) A_{o}^{(0)}
\end{aligned}
$$

The boundary condition at $x=0$ on equation (81) is given by matching with the inner solution. As before, we define the inner variable by $x=\epsilon X$. In the inner region (77) becomes

$$
\begin{aligned}
\frac{\partial H_{1}}{\partial X}=\epsilon \frac{\partial \phi_{1}}{\partial y}+\epsilon \frac{\partial A_{1}}{\partial t}+\frac{i \epsilon^{2}}{2}\left(f \frac{\partial \Psi_{1}}{\partial y}\right. & \left.-f \frac{\partial \Psi_{1}^{*}}{\partial y}\right) \\
& +\epsilon f^{2} A_{1}+\epsilon f Q\left(\Psi_{1}+\Psi_{1}^{*}\right)
\end{aligned}
$$


Since $H_{1}=0$ at $X=0$, we see that at leading order $H_{i}^{(0)}=0$. Thus the boundary conditions on (81) are

$$
\begin{aligned}
\frac{\partial A_{o}^{(0)}}{\partial x}(0, y) & =0, \\
A_{o}^{(0)} & \rightarrow 0 \quad \text { as } x \rightarrow \infty .
\end{aligned}
$$

The normal modes are given by $A_{o}^{(0)}=A(x) \sin k y$, where

$$
\frac{\partial^{2} A}{\partial x^{2}}=\left(k^{2}+\sigma^{(0)}+\left(1-3\left(Q_{o}^{(0)}\right)^{2}\right)\right) A .
$$

Hence

$$
\sigma^{(0)}=\sigma_{1 d}^{(0)}-k^{2}
$$

where $\sigma_{1 d}^{(0)}$ are the eigenvalues of the one-dimensional perturbation, all negative for $H<1 / \sqrt{2}$. Hence the superconducting solution is stable to long-wavelength perturbations for fields $H<1 / \sqrt{2}$.

We now consider the stability of short-wavelength perturbations. We consider a short timescale by setting $t=\epsilon^{2} T$ and perform a multiple-scales analysis by introducing a new lengthscale to the outer problem. We set

$$
x=\epsilon X, \quad y=\epsilon Y .
$$

We consider a perturbation of the steady-state solution of the form

$$
\begin{aligned}
\Psi & =f(x)+\delta \Psi_{1}(x, y, X, Y, T) \\
A & =Q(x)+\delta A_{1}(x, y, X, Y, T) \\
\phi & =\delta \phi_{1}(x, y, X, Y, T)
\end{aligned}
$$

Substituting into equations (1), (2) and linearising in $\delta$ yield

$$
\begin{aligned}
&-\alpha \frac{\partial \Psi_{1}}{\partial T}-\alpha \epsilon i f \phi_{1}+\frac{\partial^{2} \Psi_{1}}{\partial X^{2}}+\frac{\partial^{2} \Psi_{1}}{\partial Y^{2}}+2 \epsilon \frac{\partial^{2} \Psi_{1}}{\partial x \partial X}+2 \epsilon \frac{\partial^{2} \Psi_{1}}{\partial y \partial Y}+\epsilon^{2} \frac{\partial^{2} \Psi_{1}}{\partial x^{2}}+\epsilon^{2} \frac{\partial^{2} \Psi_{1}}{\partial y^{2}} \\
&=-2 i Q \frac{\partial \Psi_{1}}{\partial Y}-2 i \epsilon Q \frac{\partial \Psi_{1}}{\partial y}-i f \frac{\partial A_{1}}{\partial Y}-i \epsilon f \frac{\partial A_{1}}{\partial y} \\
&+2 A_{1} Q f+Q^{2} \Psi_{1}+f^{2}\left(2 \Psi_{1}+\Psi_{1}^{*}\right)-\Psi_{1}, \\
&-\frac{1}{\epsilon^{2}} \frac{\partial^{2} A_{1}}{\partial X \partial Y}-\frac{1}{\epsilon} \frac{\partial^{2} A_{1}}{\partial X \partial y}-\frac{1}{\epsilon} \frac{\partial^{2} A_{1}}{\partial x \partial Y}-\frac{\partial^{2} A_{1}}{\partial x \partial y} \quad \frac{1}{\epsilon} \frac{\partial \phi_{1}}{\partial X}+\frac{\partial \phi_{1}}{\partial x}+\frac{i}{2}\left(f \frac{\partial \Psi_{1}}{\partial X}-f \frac{\partial \Psi_{1}^{*}}{\partial X}\right)+\frac{i \epsilon}{2}\left(f \frac{\partial \Psi_{1}}{\partial x}-f \frac{\partial \Psi_{1}^{*}}{\partial x}+\Psi_{1}^{*} \frac{d f}{d x}-\Psi_{1} \frac{d f}{d x}\right), \\
& \frac{1}{\epsilon^{2}} \frac{\partial^{2} A_{1}}{\partial X^{2}}+\frac{1}{\epsilon} \frac{\partial^{2} A_{1}}{\partial x \partial X}+\frac{\partial^{2} A_{1}}{\partial x^{2}}=\frac{1}{\epsilon} \frac{\partial \phi_{1}}{\partial Y}+\frac{\partial \phi_{1}}{\partial y}+\frac{1}{\epsilon^{2}} \frac{\partial A_{1}}{\partial T}+\frac{i}{2}\left(f \frac{\partial \Psi_{1}}{\partial Y}-f \frac{\partial \Psi_{1}^{*}}{\partial Y}\right) \\
& \quad+\frac{i \epsilon}{2}\left(f \frac{\partial \Psi_{1}}{\partial y}-f \frac{\partial \Psi_{1}^{*}}{\partial y}\right)+f^{2} A_{1}+f Q\left(\Psi_{1}+\Psi_{1}^{*}\right) .
\end{aligned}
$$

We expand in powers of $\epsilon$ as before (with $\phi_{1} \sim \epsilon^{-1} \phi_{1}^{(0)}+\cdots$ ) to give at leading order 
(on dropping the 1's)

$$
\begin{aligned}
\frac{\partial^{2} \Psi^{(0)}}{\partial X^{2}}+\frac{\partial^{2} \Psi^{(0)}}{\partial Y^{2}}= & \alpha \frac{\partial \Psi^{(0)}}{\partial T}+\alpha i f^{(0)} \phi^{(0)}-2 i Q^{(0)} \frac{\partial \Psi^{(0)}}{\partial Y}-i f^{(0)} \frac{\partial A^{(0)}}{\partial Y} \\
& +2 A^{(0)} Q^{(0)} f^{(0)}+\left(Q^{(0)}\right)^{2} \Psi^{(0)} \\
& +\left(f^{(0)}\right)^{2}\left(2 \Psi^{(0)}+\Psi^{(0) *}\right)-\Psi^{(0)} \\
& -\frac{\partial^{2} A^{(0)}}{\partial X \partial Y}=\frac{\partial \phi^{(0)}}{\partial X}, \\
& \frac{\partial^{2} A^{(0)}}{\partial X^{2}}=\frac{\partial \phi^{(0)}}{\partial Y}+\frac{\partial A^{(0)}}{\partial T} .
\end{aligned}
$$

Hence

$$
\begin{aligned}
\phi^{(0)} & =\frac{\partial A^{(0)}}{\partial Y}, \\
\frac{\partial^{2} A^{(0)}}{\partial X^{2}}+\frac{\partial^{2} A^{(0)}}{\partial Y^{2}} & =\frac{\partial A^{(0)}}{\partial T} .
\end{aligned}
$$

Thus $A^{(0)}$ simply diffuses back to its preperturbed position and is stable. We check the stability of $\Psi$ by setting $A^{(0)}=\phi^{(0)}=0$. This corresponds either to considering modes in which the initial perturbation of $A$ and $\phi$ is small or to a situation in which $\alpha$ is large so that $A$ relaxes instantly on the timescale of variations in $\Psi$. The normal modes of equation (87) are then given by

$$
\Psi^{(0)}=e^{\sigma T} \cos l X(u \sin k Y+i v \cos k Y)
$$

where

$$
\begin{aligned}
\left(\alpha \sigma+k^{2}+l^{2}+2\left(f^{(0)}\right)^{2}\right) u-2 k Q^{(0)} v & =0 \\
2 k Q^{(0)} u-\left(\alpha \sigma+k^{2}+l^{2}\right) v & =0 .
\end{aligned}
$$

In order for a nonzero solution $(u, v)$ to exist we have the following dispersion relation:

$$
\alpha \sigma=-l^{2}-k^{2}-\left(f^{(0)}\right)^{2} \pm \sqrt{\left(f^{(0)}\right)^{4}+4 k^{2}\left(Q^{(0)}\right)^{2}} .
$$

Note that $\sigma$ depends on $x$ since $f^{(0)}$ and $Q^{(0)}$ do. Noting that $\left(f^{(0)}\right)^{2}=1-\left(Q^{(0)}\right)^{2}$ we find that there are modes giving positive values of $\sigma$ whenever $\left(Q^{(0)}\right)^{2}>1 / 3$. Since $\left(Q^{(0)}\right)^{2}$ is monotonic decreasing in $x$, we see that the instability will occur first at the boundary of the sample. In the boundary layer

$$
Q^{(0)}=b=\frac{2 \sqrt{2} a}{1+a^{2}},
$$

where $a$ is related to the applied field $H_{0}$ by (29). Thus we can calculate the field at which the instability first occurs, being the field at which $b^{2}=1 / 3$. We find

$$
H_{s h}=\frac{1}{3} \sqrt{\frac{5}{2}}=\frac{\sqrt{5}}{3} H_{c} \approx 0.745 H_{c} .
$$

We note that this result has been arrived at independently by Neu, via a boundary layer analysis [22]. 
Remark. We have shown that the superconducting solution becomes unstable at a certain value of the magnetic field, which we then define as the superheating magnetic field. We have not shown that this instability leads to the formation of vortices and the mixed state, though we would conjecture that it does. This remains an interesting open problem.

Remark. Bean and Livingston calculate the superheating field by considering the energy of a vortex line near a sample surface. They find that for distances close to the surface the energy increases with increasing distance, while for distances further away the energy decreases with increasing distance. Thus, they conclude, a vortex placed sufficiently close to the sample surface will be sucked out of the surface, while one far enough away will move into the interior of the sample. This is the "surface barrier" to vortex penetration. The superheating field that they calculate is the magnetic field at which the critical distance from the boundary is of the same order as the vortex core. The actual field obtained depends upon the details of the vortex core; they use a "cutoff" calculation which leads to a value of $H_{c} / \sqrt{2}$. This differs from the present calculation by the factor of $\sqrt{10 / 9}$, or 1.05 .

This definition of the superheating field is unsatisfactory for the following reasons. First, the true value of the superheating field can only be calculated by a careful analysis of a vortex whose distance from the boundary is the same order as its core radius (i.e., $O(1 / \kappa)$ ). The arguments used by Bean and Livingston can only give the order of magnitude of the superheating field. Second, a vortex near the boundary is a major perturbation to the superconducting solution, and it may be that the superconducting solution is locally stable, even though any vortices that happened to be generated would move into the sample.

An analysis of the type indicated above is needed, though, to show that any vortices generated by the instability we have found do actually make their way into the sample. This also remains an interesting open problem.

Remark. Often in the limit as $\kappa \rightarrow \infty$ in the Ginzburg-Landau equations a different scaling of the parameter $\alpha$ is chosen, which corresponds to the present $\alpha \sim \epsilon^{-2}$. This means that the timescale for relaxation of the magnetic field is much shorter than the timescale for relaxation of the order parameter, rather than the two timescales being comparable as above. Adopting this different scaling of $\alpha$ makes the long-wave stability calculation a little more tedious, though it does not alter the result. The short-wave stability calculation is the same, since the magnetic field simply relaxes instantly on the relevant timescale, as indicated above.

Remark. Note that the stability of long-wavelength perturbations is governed by the time derivative of the potential $A$, whereas the stability of short-wavelength perturbations is governed by the time derivative of the order parameter $\Psi$. This is because $A$ varies on an order one scale, while $\Psi$ can vary on an $O(1 / \kappa)$ scale. For long-wave perturbations $A$ is the dominant variable and the solution is stable, while for short-wavelength perturbations, once $A$ relaxes, $\Psi$ has a chance to dominate and generate the instability. Note also that when $k=0$, so that the perturbation is a function of $x$ only, then $\sigma<0$. These modes were not accounted for in our earlier stability analysis in one dimension. However, since they are decaying, the conclusions we came to still hold.

5. Superheating fields for sample geometries other than the slab. Since the instability mentioned above occurs on an $O(\epsilon)$ lengthscale and not an $O(1)$ lengthscale, we can now calculate the superheating field for any specimen shape in which 
the boundary varies slowly on the inner lengthscale. In such cases the stability is determined simply by the magnitude of the outer solution.

Because of the gauge-invariance property (6), it is not really the vector potential $\boldsymbol{A}$ but the gauge-invariant potential $\boldsymbol{Q}=\boldsymbol{A}-\epsilon \nabla \chi$, where $\chi$ is the phase of the order parameter $\Psi$, which is important. For the halfspace $\chi=0$, but in general it may not be.

If we solve the outer problem for $\boldsymbol{Q}$, we know that the solution will be unstable at any point at which the magnitude of $\boldsymbol{Q}$ is greater than $1 / \sqrt{3}$, without needing to do the stability calculation again. In the steady state, the outer problem is given by

$$
\begin{array}{rlrl}
-(\operatorname{curl})^{2} \boldsymbol{Q} & =\left(1-|\boldsymbol{Q}|^{2}\right) \boldsymbol{Q} & \text { in } \Omega, \\
\boldsymbol{Q} \cdot \boldsymbol{n} & =0 & \text { on } \partial \Omega, & \\
\operatorname{curl} \boldsymbol{Q} & =H_{0} \hat{\boldsymbol{z}} & & \text { on } \partial \Omega .
\end{array}
$$

Two geometries in which this problem reduces to an ordinary differential equation are the film $-d<x<d$, in which case $\boldsymbol{Q}=(0, Q(x), 0)$,

$$
\begin{aligned}
Q^{\prime \prime} & =Q-Q^{3}, \\
Q^{\prime}( \pm d) & =H_{0},
\end{aligned}
$$

where $I \equiv d / d x$, and the circular cylinder $r<R$, in which case $\boldsymbol{Q}=Q(r) \boldsymbol{\theta}$,

$$
\begin{aligned}
Q^{\prime \prime}+\frac{Q^{\prime}}{r}-\frac{Q}{r^{2}} & =Q-Q^{3}, \\
Q^{\prime}(R)+\frac{Q(R)}{R} & =H_{0}, \\
Q(0) & \text { bounded, }
\end{aligned}
$$

where $I \equiv d / d r$ and $\boldsymbol{\theta}$ is the unit vector in the azimuthal direction. The superheating fields for these two geometries are shown in Figs. 4 and 5, respectively.

We can get an idea of how the shape of the boundary affects the superheating field by considering perturbations of the slab and cylindrical geometries. We consider here a halfspace with a slowly varying, small-amplitude sine-wave perturbation of the boundary. Here the wavelength of the perturbation has to be $\gg \epsilon$ (so that the boundary is still planar in the boundary layer expansion). The amplitude of the perturbation should be $\ll 1$ but $\gg \epsilon$, since we will calculate a series expansion in powers of it while neglecting terms of order $\epsilon$ in our original expansion.

We consider the domain $x>\delta \sin k y, \epsilon \ll \delta \ll 1$. The equations are then

$$
\begin{gathered}
-\frac{\partial}{\partial y}\left(\frac{\partial Q_{2}}{\partial x}-\frac{\partial Q_{1}}{\partial y}\right)=\left(1-Q_{1}^{2}-Q_{2}^{2}\right) Q_{1}, \\
\frac{\partial}{\partial x}\left(\frac{\partial Q_{2}}{\partial x}-\frac{\partial Q_{1}}{\partial y}\right)=\left(1-Q_{1}^{2}-Q_{2}^{2}\right) Q_{2},
\end{gathered}
$$

with boundary conditions

$$
\begin{aligned}
Q_{1}(\delta \sin (k y), y)-k \delta \cos (k y) Q_{2}(\delta \sin (k y), y) & =0 \\
\frac{\partial Q_{2}}{\partial x}(\delta \sin (k y), y)-\frac{\partial Q_{1}}{\partial y}(\delta \sin (k y), y) & =H_{0}
\end{aligned}
$$




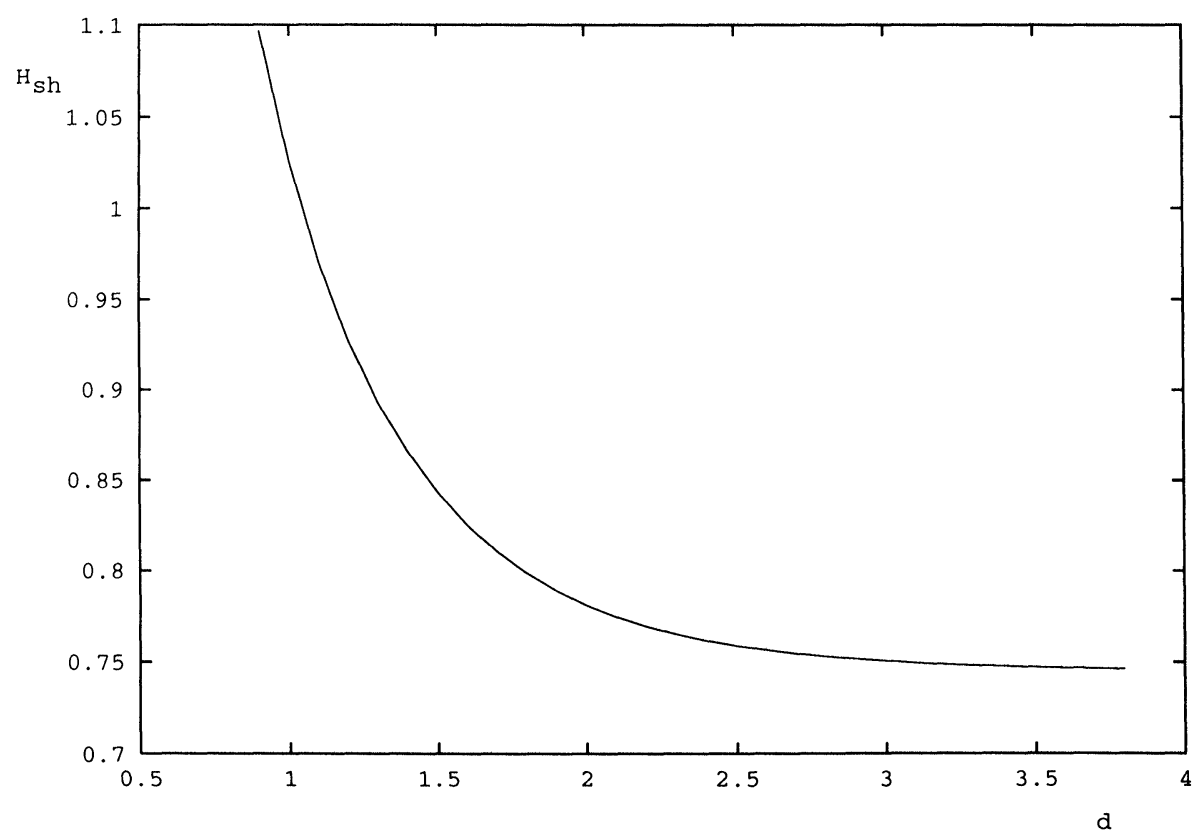

FIG. 4. The superheating field $H_{s h}$ as a function of thickness for a slab of size $2 d$.

Expanding $Q_{1}$ and $Q_{2}$ in powers of $\delta$ we find

$$
\begin{aligned}
& Q_{1}=\delta Q_{1}^{(1)}+\cdots \\
& Q_{2}=Q+\delta Q_{2}^{(1)}+\cdots,
\end{aligned}
$$

where $Q$ is the previously found solution for a halfspace given by $(25)$ and $Q_{1}^{(1)}$ and $Q_{2}^{(1)}$ satisfy

$$
\begin{gathered}
-\frac{\partial^{2} Q_{2}^{(1)}}{\partial x \partial y}+\frac{\partial^{2} Q_{1}^{(1)}}{\partial y^{2}}=\left(1-Q^{2}\right) Q_{1}^{(1)} \\
\frac{\partial^{2} Q_{2}^{(1)}}{\partial x^{2}}-\frac{\partial^{2} Q_{1}^{(1)}}{\partial x \partial y}=\left(1-3 Q^{2}\right) Q_{2}^{(1)}
\end{gathered}
$$

with boundary conditions

$$
Q_{1}^{(1)}(0, y)=k Q(0) \cos k y
$$

$$
\frac{\partial Q_{2}^{(1)}}{\partial x}(0, y)-\frac{\partial Q_{1}^{(1)}}{\partial y}(0, y)=-\frac{\partial^{2} Q}{\partial x^{2}}(0) \sin k y
$$

Seeking a solution of the form

$$
\begin{aligned}
& Q_{1}^{(1)}=f(x) \cos k y, \\
& Q_{2}^{(1)}=g(x) \sin k y,
\end{aligned}
$$

we have

$$
\begin{aligned}
-k g^{\prime}-k^{2} f & =\left(1-Q^{2}\right) f, \\
g^{\prime \prime}+k f^{\prime} & =\left(1-3 Q^{2}\right) g,
\end{aligned}
$$




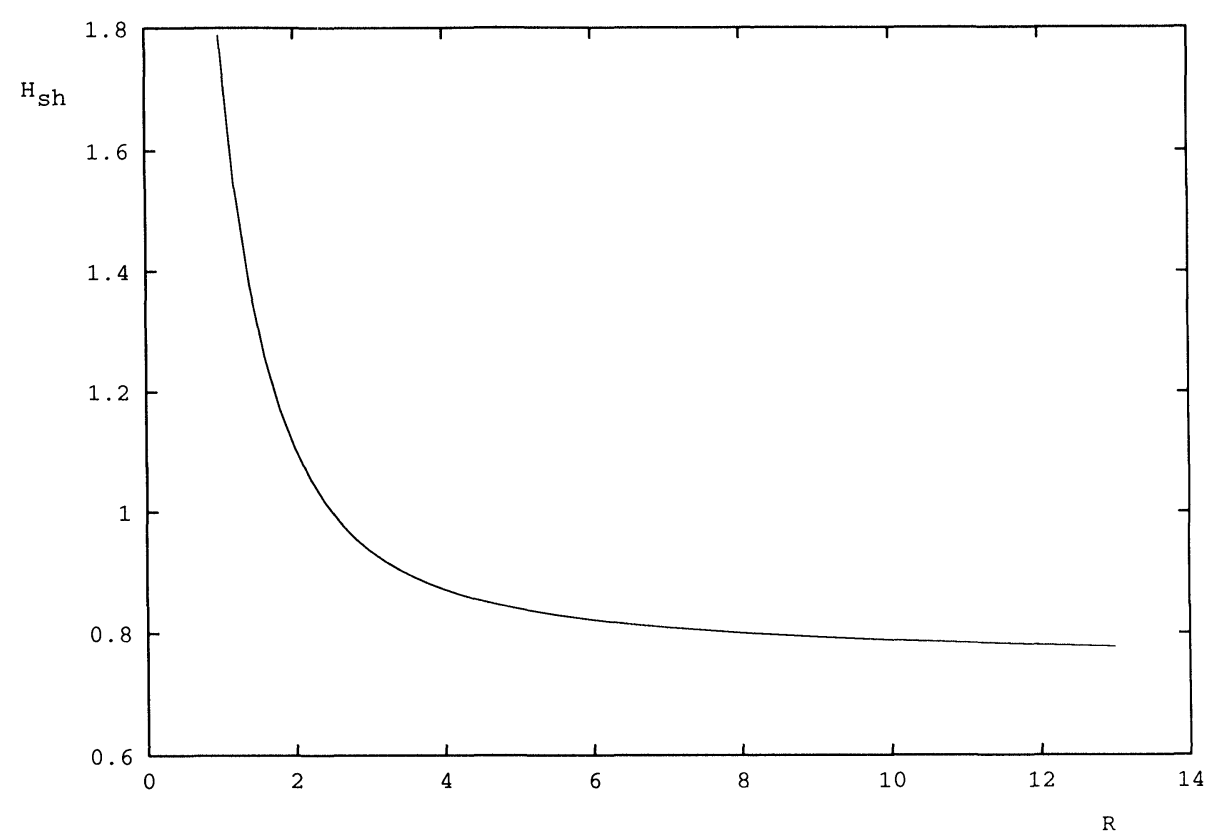

FIG. 5. The superheating field $H_{\text {sh }}$ as a function of radius $R$ for a cylinder.

with boundary conditions

$$
\begin{aligned}
f(0) & =k Q(0), \\
g^{\prime}(0) & =-k^{2} Q(0)-Q^{\prime \prime}(0)=-Q(0)\left\{1+k^{2}-Q(0)^{2}\right\},
\end{aligned}
$$

where $I \equiv d / d x$. The remaining boundary conditions come from the constraint that $f$ and $g$ must be bounded as $x \rightarrow \infty$, which implies $f(\infty)=g(\infty)=0$. We are interested in $|\boldsymbol{Q}|$. We have

$$
|\boldsymbol{Q}|^{2}=Q(x)^{2}+2 \delta Q(x) g(x) \sin k y+O\left(\delta^{2}\right) .
$$

Evaluating this on the boundary we find

$$
|\boldsymbol{Q}|^{2}(\delta \sin k y, y)=Q(0)^{2}+2 \delta Q(0)\left[Q^{\prime}(0)+g(0)\right] \sin k y+O\left(\delta^{2}\right) .
$$

Thus we are interested primarily in the quantity $g+Q^{\prime}=w$, say. Eliminating $f$ and using (24) we find

$$
\begin{aligned}
w^{\prime \prime}-k^{2}\left(\frac{w^{\prime}}{1+k^{2}-Q^{2}}\right)^{\prime} & +k^{2}\left(\frac{Q\left(1-Q^{2}\right)}{1+k^{2}-Q^{2}}\right)^{\prime}=\left(1-3 Q^{2}\right) w \\
w^{\prime}(0) & =-k^{2} Q(0), \\
w & \rightarrow 0 \quad \text { as } x \rightarrow \infty
\end{aligned}
$$

Let us consider the case in which $Q$ is exactly on the point of instability, i.e., $Q(0)=$ $-1 / \sqrt{3}$. It is then of interest to see whether the instability occurs at the troughs or peaks of the boundary perturbation. This will depend on whether $w(0)$ is greater 
than or less than zero. It is not difficult to show that $g(0)$ must be less than zero. However, $Q^{\prime}(0)$ is positive, and so we need to work harder to determine the sign of $w$.

Even in this simplified form the problem (118)-(120) is still difficult. We simplify it further by considering the limits of small and large wavenumber $k$.

Small wavenumber. For small $k$ we expand

$$
w=w_{0}+k^{2} w_{1}+\cdots
$$

Then at leading order

$$
\begin{aligned}
w_{0}^{\prime \prime} & =\left(1-3 Q^{2}\right) w_{0}, \\
w_{0}^{\prime}(0) & =0, \\
w_{0} & \rightarrow 0 \quad \text { as } x \rightarrow 0 .
\end{aligned}
$$

Hence $w_{0}=0$. At first order we find

$$
\begin{aligned}
w_{1}^{\prime \prime}+Q^{\prime} & =\left(1-3 Q^{2}\right) w_{1}, \\
w_{1}^{\prime}(0) & =-Q(0), \\
w_{1} & \rightarrow 0 \quad \text { as } x \rightarrow 0 .
\end{aligned}
$$

We define

$$
\phi=-\int_{0}^{x} Q(x) d x .
$$

Then $\phi>0, \phi^{\prime}>0, \phi^{\prime \prime}<0$, for $x>0$. Letting $v=w_{1}-\phi$, we have

$$
\begin{aligned}
v^{\prime \prime} & =\left(1-3 Q^{2}\right) w_{1}, \\
v^{\prime}(0) & =0, \\
v^{\prime} & \rightarrow 0 \quad \text { as } x \rightarrow \infty .
\end{aligned}
$$

Now suppose that $w_{1}(0)=\alpha>0$. Then $v(0)=\alpha>0, v^{\prime}(0)=0, v^{\prime \prime}(0)=0$, $v^{\prime \prime \prime}(0)>0$. Thus $v$ is increasing initially, and we have $v^{\prime}>0$ in a neighbourhood of the origin. Suppose there is a first point $x_{0}$ such that $v^{\prime \prime}\left(x_{0}\right)=0$. Then $w_{1}\left(x_{0}\right)=0$, which implies $v\left(x_{0}\right)=-\phi\left(x_{0}\right)<0$, contradicting the minimality of $x_{0}$. Hence $v^{\prime \prime}>0$ for all $x$, and $v$ cannot satisfy the boundary condition at infinity. Thus $\alpha$ cannot be positive; i.e., $w_{1}(0) \leq 0$. From (117) we see that this implies that $|\boldsymbol{Q}|$ is increased in places on the boundary where sin $k y$ is positive (since $Q(0)<0$ ), and therefore it is at these points that the instability will first occur, as shown in Fig. 6. Note that the effect on the superheating field of the boundary perturbation is $O\left(k^{2}\right)$ for small $k$.

Large wavenumber. The limit of large wavenumber in (118)-(120) is a singular perturbation problem, since the coefficient of $w^{\prime \prime}$ tends to zero as $k \rightarrow \infty$. Thus we expect that there will be a boundary layer at $x=0$. Indeed, if we naively let $k$ tend to infinity, then the leading-order behaviour of $w$ is

$$
w=\frac{\left(Q\left(1-Q^{2}\right)\right)^{\prime}}{1-3 Q^{2}}
$$

which does not satisfy the boundary condition at $x=0$. 


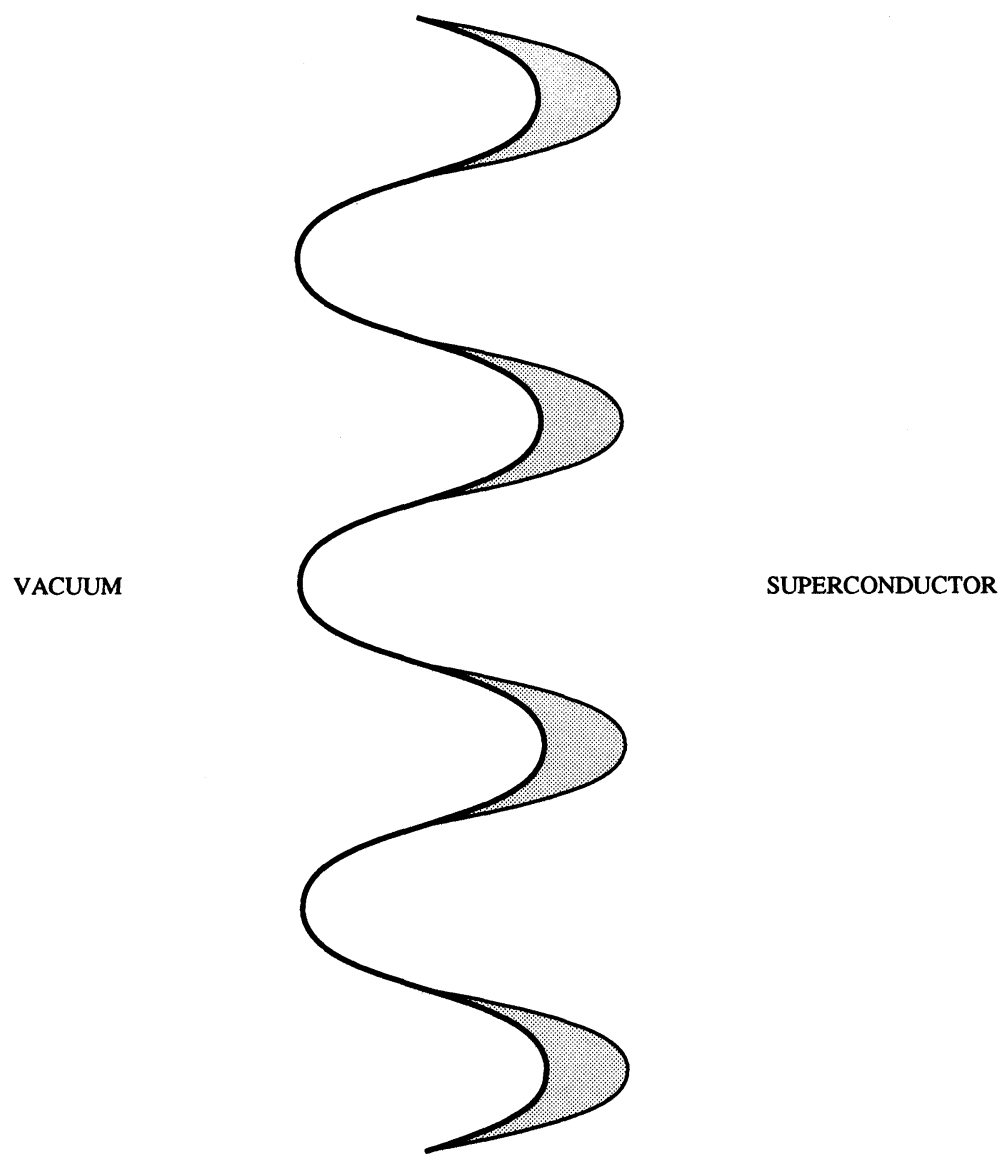

FIG. 6. Diagram showing the region of first instability for a sine-wave perturbation of a halfspace.

The expansion

$$
Q \sim-\frac{1}{\sqrt{3}}+\frac{\sqrt{5}}{3 \sqrt{2}} x+\cdots \quad \text { as } x \rightarrow 0
$$

motivates the following scalings in the boundary layer. We define the inner coordinate by $x=X k^{-2 / 3}$ and let $w=k^{4 / 3} W$. Then the leading-order behaviour of $W$ is given by

$$
\begin{aligned}
W^{\prime \prime} & =\sqrt{\frac{15}{2}} X W, \\
W^{\prime}(0) & =\frac{1}{\sqrt{3}}, \\
W & \rightarrow 0 \quad \text { as } X \rightarrow \infty .
\end{aligned}
$$

Hence

$$
W=\left(\frac{2}{15}\right)^{1 / 6} \frac{1}{\sqrt{3} \mathrm{Ai}^{\prime}(0)} \mathrm{Ai}\left(\left(\frac{15}{2}\right)^{1 / 6} X\right)
$$


where $\mathrm{Ai}$ is the Airy function. In particular, since $\operatorname{Ai}(0)>0$ and $\operatorname{Ai}^{\prime}(0)<0$, we see that $W(0)<0$. Thus, as for small wavenumber, $|\boldsymbol{Q}|$ is increased in places on the boundary where $\sin k y$ is positive. Thus for large wavenumber the instability will also first occur at the places shown in Fig. 6 . Note that the effect on the superheating field of the boundary perturbation is $O\left(k^{4 / 3}\right)$ for large $k$. Thus small-wavelength perturbations have a much stronger effect on the superheating field than long-wavelength perturbations.

Figure 7 shows a numerical calculation of $w(0)$ plotted against wavenumber $k$.

Moving back to the general situation, we can reformulate the outer problem (92)(94) in terms of the variables which are of primary interest, namely, the magnetic field $H$ and the square magnitude of the potential $\boldsymbol{Q}$, which we will call $u$. If $\boldsymbol{Q}=\left(Q_{1}, Q_{2}\right)$ then $H=\partial Q_{2} / \partial x-\partial Q_{1} / \partial y, u=Q_{1}^{2}+Q_{2}^{2}$, and (92)-(94) become

$$
\begin{aligned}
-\frac{\partial H}{\partial y} & =(1-u) Q_{1} \text { in } \Omega, \\
\frac{\partial H}{\partial x} & =(1-u) Q_{2} \text { in } \Omega, \\
H & =H_{0} \text { on } \partial \Omega .
\end{aligned}
$$

(Note that (93) follows from (92) and (94) since $H_{0}$ is constant, and is therefore superfluous.) Hence

$$
\begin{aligned}
H & =\nabla \cdot\left(\frac{\nabla H}{1-u}\right) \quad \text { in } \Omega, \\
u(1-u)^{2} & =|\nabla H|^{2} \quad \text { in } \Omega, \\
H & =H_{0} \quad \text { on } \partial \Omega .
\end{aligned}
$$

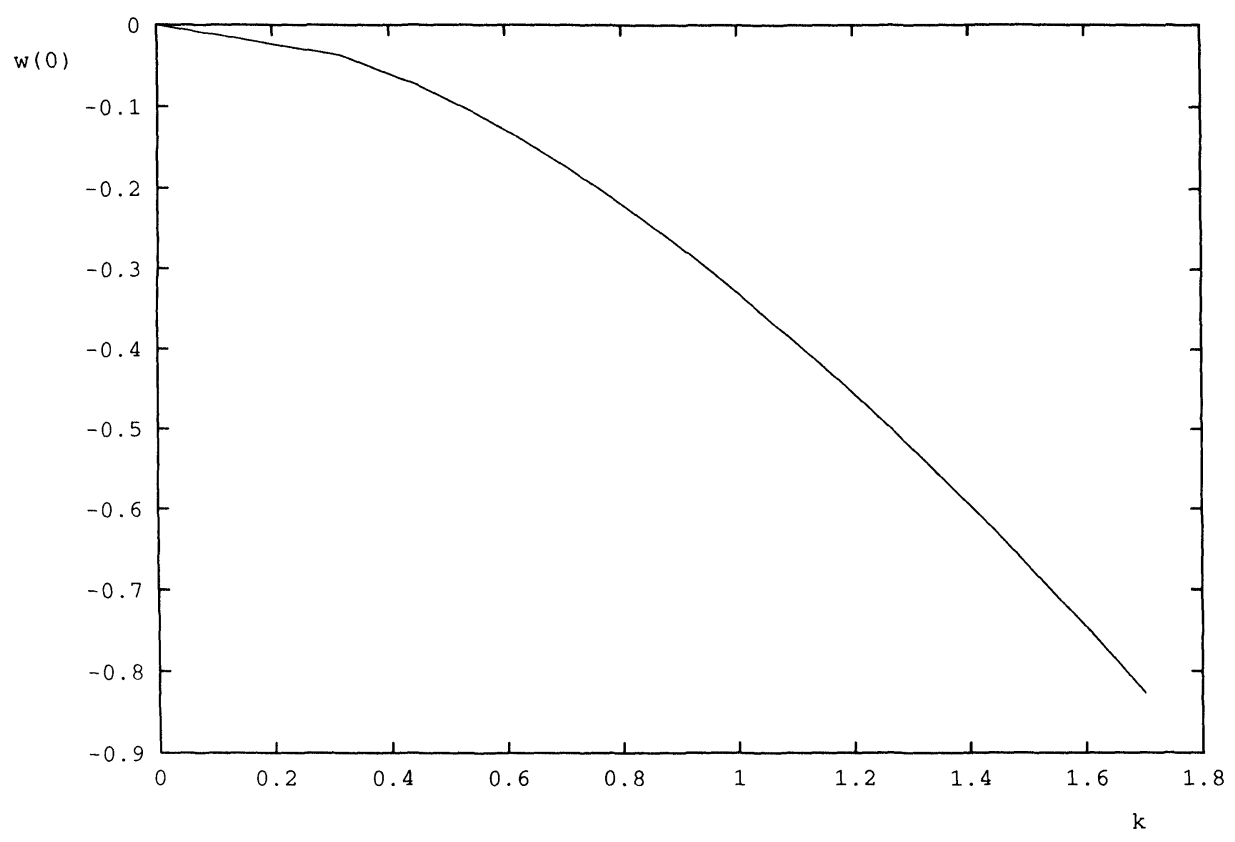

FIG. 7. $w(0)$ as a function of $k$. 
Questions of interest here are the value of $H_{0}$ and the position in the sample at which $u$ first becomes equal to $1 / 3$. Is the first point at which $u$ equals $1 / 3$ always on the boundary? Since $1 / 3$ is relatively small compared to unity, one way of approaching this problem would be to perform an asymptotic expansion in powers $u$. The leadingorder magnetic field would then satisfy

$$
\begin{aligned}
\nabla^{2} H^{(0)}-H^{(0)} & =0 \text { in } \Omega, \\
H^{(0)} & =H_{0} \text { on } \partial \Omega .
\end{aligned}
$$

The leading-order $u$ is then given by $u^{(0)}=\left|\nabla H^{(0)}\right|^{2}$. The error in the leadingorder solution should be of the order of $\left(u^{(0)}\right)^{2}$, or about $10 \%$. Of course, it can be improved upon if we compute higher terms in the expansion. These terms will each satisfy inhomogeneous versions of the modified Helmholz equation, with known right-hand sides.

The leading-order approximation for a slab is

$$
H^{(0)}=H_{0} e^{-x}
$$

This gives the leading-order superheating field as $H_{s h}=1 / \sqrt{3}=0.577$, which is about $10 \%$ larger than the true value of 0.527 .

6. Comparison with experiment. We compare the superheating field $H_{s h}=$ $0.745 H_{c}$ calculated in $\S 4$ with the available experimental data.

Boato et al. [5] perform superheating experiments by applying an axial alternating magnetic field to a cylindrical sample with radius approximately equal to $4 \lambda$. They find that the field at which the first vortex enters the sample is 190 Gauss. The alloy filament they use has a $\kappa$ value of about 8, and a thermodynamic field of 275 Gauss. Thus they find $H_{s h}=0.69 H_{c}$.

De Blois and De Sorbo [4] use a larger sample with $\kappa \approx 4, H_{c}=1360$ Oe. They find a superheating fields varying along the length of the specimen from 750 Oe to 1400 Oe, corresponding to $0.55 H_{c}$ to $1.03 H_{c}$. In another sample with $\kappa \approx 4, H_{c}=310$ Oe. Then the average superheating field was 245 Oe and, after the next electropolish, $235 \mathrm{Oe}$, corresponding to $0.79 \mathrm{H}_{c}$ and $0.76 \mathrm{H}_{c}$, respectively.

Quantitative experimental data for other geometries is harder to come by, but there does seem to be at least qualitative agreement with the theory presented. For example, for rectangular samples vortices were first nucleated in the middle of the longest side, while for more irregular shapes interior angles were the preferred nucleation sites [24].

7. Conclusion. We have examined the solution of the Ginzburg-Landau equations for a halfspace in the limit as the Ginzburg-Landau parameter $\kappa \rightarrow \infty$. We find that there is a folding over of the response diagram so that as the applied magnetic field is increased beyond the value at the nose the solution must jump to another branch. This is the superheating field calculated by de Gennes [14].

An examination of the linear stability of the solution with respect to one-dimensional perturbations revealed that the higher of the two solution branches was indeed stable until the nose was reached, while the lower of the two solution branches was unstable. However, on examining the linear stability of the solution with respect to two-dimensional perturbations we found that it became unstable to short-wavelength perturbations at a lower value of the applied magnetic field, namely, $H_{s h}=\sqrt{5} H_{c} / 3$, which is therefore the true superheating field. This value agrees with that obtained 
by Kramer in [19] but is different from that obtained by Bean and Livingston in [3] (although the difference is only $5 \%$ ). It agrees well with experimental observations.

Finally, we examined the superheating field for geometries other than a halfspace. We found that for sine-wave perturbations of a halfspace, the instability first sets in at the troughs rather than at the peaks of the perturbation, i.e., at points on the boundary with the largest negative curvature. The general problem of calculating the superheating fields for an arbitrary domain (137)-(139) remains an interesting open question.

\section{REFERENCES}

[1] A. A. Abrikosov, On the magnetic properties of superconductors of the second group, Soviet Phys. J.E.T.P., 5 (1957), pp. 1174-1182.

[2] J. Bardeen, L. N. Cooper, ANd J. R. Schreiffer, Thoery of superconductivity, Phys. Rev., 108 (1957), p. 1175-1204.

[3] C. P. BeAn And J. D. Livingston, Surface barrier in type II superconductors, Phys. Rev. Lett., 12 (1964), pp. 14-16.

[4] R. W. DE BLOIS AND W. DE SoRBo, Surface barrier in type II superconductors, Phys. Rev. Lett., 12 (1964), pp. 499-501.

[5] G. Boato, G. Gallinaro, And C. Rizzuto, Direct evidence for quantized fluz threads in type II superconductors, Solid State Comm., 3 (1965), pp. 173-176.

[6] C. Bolley AND B. HelfFeR, Rigorous results on Ginzburg-Landau models in a film submitted to an exterior parallel magnetic field. Ecole Central de Nantes, 1994, preprint.

[7] S. J. Chapman, Asymptotic analysis of the Ginzburg-Landau model of superconductivity: Reduction to a free boundary model, Quart. Appl. Math., (1995), to appear.

[8] S. J. Chapman, Nucleation of superconductivity in decreasing fields I, European J. Appl. Math., 5 (1994), pp. 449-468.

[9] S. J. Chapman, Nucleation of superconductivity in decreasing fields II, European J. Appl. Math., 5 (1994), pp. 469-494.

[10] S. J. Chapman, S. D. Howison, And J. R. OCKendon, Macroscopic models of superconductivity, SIAM Rev., 34, (1992), pp. 529-560.

[11] Q. Du, M. D. Gunzburger, And J. S. Peterson, Analysis and approximation of the GinzburgLandau model of superconductivity, SIAM Rev., 34 (1992), pp. 54-81.

[12] U. EsSMANN AND H. TRÄUBLE, The direct observation of individual flux lines in type-II superconductors, Phys. Lett., A24 (1967), p. 526.

[13] H. J. Fink, Delayed flux entry into type II superconductors, Phys. Lett. L, 20 (1966), pp. 356357.

[14] P. G. DE Gennes, Vortex nucleation in type II superconductors, Solid State Comm., 3 (1965), pp. $127-130$.

[15] V. L. GinzBurg And L. D. LANDAU, On the theory of superconductivity, J. Experiment. Theoret. Phys., 20 (1950), p. 1064.

[16] L. P. Gor'Kov And G. M. ÉLIASHBerg, Generalisation of the Ginzburg-Landau equations for non-stationary problems in the case of alloys with paramagnetic impurities, Soviet Phys. J.E.T.P., 27 (1968), p. 328.

[17] J. B. Keller, Propagation of a magnetic field into a superconductor, Phys. Rev., 111 (1958), p. 1497.

[18] W. H. Kleiner, L. M. Roth, And S. H. Autler, Bulk solution of Ginzburg-Landau equations for type-II superconductors: Upper critical field region, Phys. Rev. A, 133 (1964), p. 1226.

[19] L. Kramer, Vortex nucleation in type-II superconductors, Phys. Lett. A, 24 (1967), p. 571.

[20] J. Matricon And D. SAInt-JAmes, Superheating fields in superconductors, Phys. Lett. A, 24 (1967), p. 241.

[21] M. H. Millman And J. B. Keller, Perturbation theory of nonlinear boundary-value problems, J. Math. Phys., 10 (1969), p. 342.

[22] J. Neu, Evolution and Creation of Vortices and Domain Walls, unpublished lecture notes.

[23] F. ODEH, Existence and bifurcation theorems for the Ginzburg-Landau equations, J. Math. Phys., 8 (1967), p. 2351.

[24] Video presentations by M. Indenbom, C. Duran, M. Jeffery, and V. Vlasko-Vlasov, Workshop on the Statics and Dynamics of Vortices in Superconductors, University of Oregon, Eugene, 1993. 